\title{
Engineering Dynamical Couplings for Quantum Thermodynamic Tasks
}

\author{
Matteo Carrega, ${ }^{1, *}$ Loris Maria Cangemi $\odot,{ }^{2,3}$ Giulio De Filippis, ${ }^{2,3,4}$ Vittorio Cataudella, ${ }^{2,3,4}$ \\ Giuliano Benenti® ${ }^{5,6,7}$ and Maura Sassetti ${ }^{8,1}$ \\ ${ }^{1}$ CNR-SPIN, Via Dodecaneso 33, Genova 16146, Italy \\ ${ }^{2}$ Dipartimento di Fisica "E. Pancini”, Università di Napoli "Federico II", Complesso di Monte S. Angelo, via \\ Cinthia, Napoli 80126, Italy \\ ${ }^{3}$ CNR-SPIN, clo Complesso di Monte S. Angelo, via Cinthia, Napoli 80126, Italy \\ ${ }^{4}$ INFN, Sezione di Napoli, Complesso Universitario di Monte S. Angelo, Napoli I-80126, Italy \\ ${ }^{5}$ Center for Nonlinear and Complex Systems, Dipartimento di Scienza e Alta Tecnologia, Università degli Studi \\ dell'Insubria, via Valleggio 11, Como 22100, Italy \\ ${ }^{6}$ Istituto Nazionale di Fisica Nucleare, Sezione di Milano, via Celoria 16, Milano 20133, Italy \\ ${ }^{7}$ NEST, Istituto Nanoscienze-CNR, Pisa I-56126, Italy \\ ${ }^{8}$ Dipartimento di Fisica, Università di Genova, Via Dodecaneso 33, Genova 16146, Italy
}

(Received 23 September 2021; accepted 18 January 2022; published 9 February 2022)

\begin{abstract}
Describing the thermodynamic properties of quantum systems far from equilibrium is challenging, in particular when the system is strongly coupled to its environment, or when memory effects cannot be neglected. Here, we address such regimes when the system-bath couplings are periodically modulated in time. We show that the couplings modulation, usually associated with a purely dissipative effect, can be suitably engineered to perform thermodynamic tasks. In particular, asymmetric couplings to two heat baths can be used to extract heat from the cold reservoir and to realize an ideal heat rectifier, where the heat current can be blocked either in the forward or in the reverse configuration by simply tuning the frequency of the couplings modulation. Interestingly, both effects take place in the low-temperature, quantum nonMarkovian regime. Our work paves the way for the use of optimal control techniques for heat engines and refrigerators working in regimes beyond standard approaches.
\end{abstract}

DOI: 10.1103/PRXQuantum.3.010323

\section{INTRODUCTION}

The development of quantum technologies [1] requires a deeper understanding of the thermodynamics of far from equilibrium quantum systems [2-13]. Questions like how to efficiently manage heat at the nanoscale [14-17], what are the ultimate bounds to the performance of heat engines [18-30] and how these are affected by coherence, entanglement, and quantum fluctuations [31-39], and what is the minimum temperature achievable in a given time in small quantum refrigerators [40-44], are vital for the construction of quantum machines. For instance, manipulating heat flows via devices like thermal switches, diodes, and transistors is essential to evacuate heat in quantum processors $[17,45,46]$, while efficient cooling is strictly

*matteo.carrega@spin.cnr.it

Published by the American Physical Society under the terms of the Creative Commons Attribution 4.0 International license. Further distribution of this work must maintain attribution to the author(s) and the published article's title, journal citation, and DOI. related to the preparation of a target state, say for a qubit, with the desired fidelity and in the shortest possible time [42,47-49].

Master equations are an invaluable tool to investigate the dynamics of open quantum systems [50,51]. On the other hand, such equations typically rely on the assumptions that the system-bath couplings are weak and the baths large enough to neglect non-Markovian effects in the system's dynamics. These approximations, which are quite natural for macroscopic systems, easily break down when considering small quantum systems. Such considerations motivate the huge effort under way in the development of methods and tools to address regimes of strong coupling and where memory effects and system-bath quantum correlations are important [13,52-62].

Here, we develop a general framework to address the above questions in periodically driven quantum systems. In particular, we focus on the work contributions related to the system-bath couplings. These terms, overlooked in standard master equation approaches, may play a relevant role, especially when dealing with cyclic processes, like in heat engines or refrigerators. Indeed, in one cycle the 
system is connected (disconnected) to (from) one or more baths several times, in a way dependent on the specific protocol. The work contribution related to the bath couplings was found to be detrimental to the efficiency of a four-stroke Otto engine [61]. This raises the question of whether this coupling work is always deleterious, as one might intuitively argue by associating some dissipation to the nonadiabatic switching on and off of the couplings.

In contrast with such a naive argument, here we show that, by periodically modulating only the system-bath couplings in a suitably engineered way, it is possible to perform fundamental quantum thermodynamic tasks. Specifically, we show that it is possible to cool a reservoir at low temperature decreasing the coupling strength, so that the zero temperature limit can be achieved only in infinite time, in agreement with Nernst's unattainability principle [40-43]. This surprising effect is possible due to the asymmetric time-dependent couplings of the system to two heat baths, and the mixing of different harmonics. We point out that, differently from the ratchet effects discussed in the literature due to modulated baths' temperatures [63,64], where heat is evacuated asymmetrically in the two reservoirs, in our case we have a refrigerator and therefore heat is extracted from the cold reservoir. Quite interestingly, this effect takes place only in the low-temperature, non-Markovian quantum regime, and it vanishes in the high-temperature, Markovian classical regime.

We also show that the same setup, with asymmetrically modulated couplings to two baths at temperatures $T_{1}$ and $T_{2}$, can be used to build a dynamically induced heat rectifier. Here large rectification can also be obtained in the classical regime, with the possibility to achieve ideal rectification, blocking of the heat flow in the forward configuration (say, with $T_{1}>T_{2}$ ). Conversely, and remarkably, the low-temperature quantum regime turns out to be more versatile. Indeed, by simply tuning the frequency of the couplings modulation, dynamical heat rectification can be obtained in both directions, eventually blocking the heat flow in either the forward $\left(T_{1}>T_{2}\right)$ or the backward $\left(T_{1}<T_{2}\right)$ configuration. Interestingly, this peculiar operating regime corresponds to that where refrigeration properties can be obtained.

The paper is organized as follows. In Sec. II we present the general setting of periodically driven system-bath couplings and the definitions and expressions of the key thermodynamic quantities. The general approach, based on the out-of-equilibrium Green function, is presented in Sec. III. Here, we solve the dynamics at long times and we evaluate the time average (over one cycle) of the heat currents. Section IV is devoted to the discussion of our main results, namely cooling, refrigeration, and rectification properties induced by the time-dependent system-bath coupling. Section V contains the summary and conclusions. Technical details can be found in several appendices.

\section{GENERAL SETTING}

\section{A. Model}

We consider a quantum system linearly coupled to a set of $N$ reservoirs as sketched in Fig. 1, described by the total Hamiltonian (hereafter we set $\hbar=k_{B}=1$ )

$$
H^{(t)}=H_{S}+\sum_{\nu=1}^{N}\left[H_{\nu}+H_{\mathrm{int}, v}^{(t)}\right] .
$$

Each bath is modeled as a collection of harmonic oscillators in the usual Caldeira-Leggett $[51,55,57,65,66]$ framework:

$$
H_{v}=\sum_{k=1}^{\infty}\left[\frac{P_{k, v}^{2}}{2 m_{k, v}}+\frac{m_{k, v} \omega_{k, v}^{2} X_{k, v}^{2}}{2}\right],
$$

where $X_{k, v}$ and $P_{k, v}$ are the position and momentum of the $k$ th oscillator of the $v$ th baths, respectively, with $m_{k, v}$ and $\omega_{k, v}$ the associated mass and frequency. This is a standard microscopic description of a thermal bath with many degrees of freedom. There are many examples of its proper and useful application. For instance, it well describes the quantum to classical behavior of Brownian motion $[51,65]$, an ohmic resistor (which has a linear electric element), and superconducting circuits $[51,67,68]$. The interaction parts are bilinear in the position operators of system and reservoirs, and are assumed time dependent in order to modulate in time their couplings, as pictorially shown in Fig. 1. They read

$$
H_{\mathrm{int}, v}^{(t)}=\sum_{k=1}^{\infty}\left\{-x g_{\nu}(t) c_{k, v} X_{k, \nu}+x^{2} g_{v}^{2}(t) \frac{c_{k, v}^{2}}{2 m_{k, \nu} \omega_{k, \nu}^{2}}\right\},
$$

where we have introduced, here and in Eq. (1), the superscript index $(t)$ to indicate the parametric time dependence for observables, related to the driven couplings [69]. In the

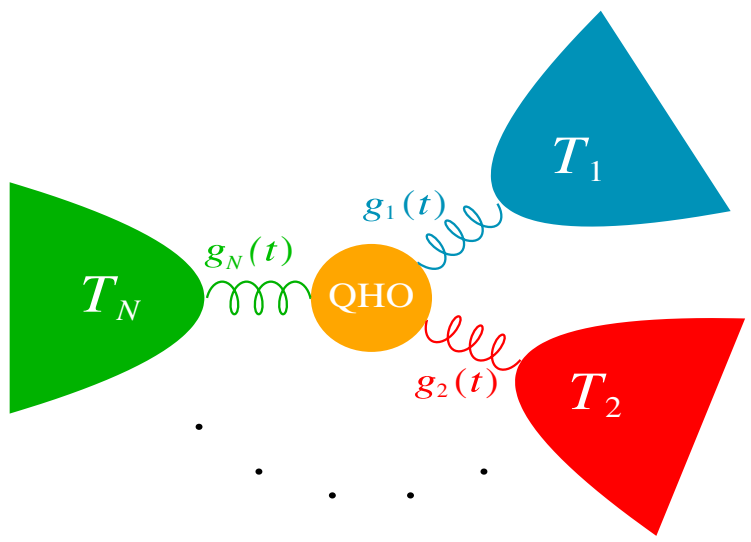

FIG. 1. Sketch of the quantum system coupled to different baths with driven system-bath couplings. 
following, we focus on cyclic processes that are governed by dimensionless and periodic time-dependent functions $g_{v}(t)=g_{v}(t+\mathcal{T})$, whose Fourier expansions read

$$
g_{\nu}(t)=\sum_{n=-\infty}^{+\infty} g_{n, \nu} e^{-i n \Omega t}, \quad \Omega=\frac{2 \pi}{\mathcal{T}}
$$

The interaction strengths are described by the parameter $c_{k, v}$ [51], and for this reason, we consider bounded functions $\left|g_{v}(t)\right| \leq 1$.

In this work, the system considered is a single quantum harmonic oscillator $(\mathrm{QHO})$, which represents a common building block for several quantum technology platforms $[66,70]$,

$$
H_{S}=\frac{p^{2}}{2 m}+\frac{1}{2} m \omega_{0}^{2} x^{2},
$$

with $m$ and $\omega_{0}$ the mass and the characteristic frequency, respectively.

At initial time $t_{0}$ the baths are assumed in their thermal equilibrium at temperatures $T_{\nu}$, with the total density matrix, describing the system plus reservoirs, written in factorized form as $\rho\left(t_{0}\right)=\rho_{S}\left(t_{0}\right) \otimes \rho_{1}\left(t_{0}\right) \otimes \cdots \otimes \rho_{N}\left(t_{0}\right)$, where $\rho_{S}\left(t_{0}\right)$ is the initial system density and

$$
\rho_{\nu}\left(t_{0}\right)=\exp \left(-H_{\nu} / T_{v}\right) / \operatorname{Tr}\left\{\exp \left(-H_{\nu} / T_{\nu}\right)\right\}
$$

is the thermal density of each bath. It is then assumed that the time-dependent couplings $g_{v}(t)$ are switched on at time $t=t_{0}^{+}$.

Using the total Hamiltonian (1), we can explicitly write the equations of motion (EOM) for the QHO operators $[x(t), p(t)]$ and for the baths oscillator operators $\left[X_{k, v}(t), P_{k, v}(t)\right]$. We have

$$
\begin{aligned}
\dot{x}(t)= & \frac{p(t)}{m}, \\
\dot{p}(t)= & -m \omega_{0}^{2} x(t) \\
& +\sum_{\nu=1}^{N} \sum_{k=1}^{\infty} g_{v}(t) c_{k, v}\left[X_{k, v}(t)-\frac{g_{v}(t) c_{k, v}}{m_{k, \nu} \omega_{k, v}^{2}} x(t)\right],
\end{aligned}
$$

and

$$
\begin{aligned}
\dot{X}_{k, v}(t) & =\frac{P_{k, v}(t)}{m_{k, v}} \\
\dot{P}_{k, v}(t) & =-m_{k, v} \omega_{k, v}^{2} X_{k, v}(t)+g_{v}(t) c_{k, v} x(t) .
\end{aligned}
$$

The solution for the baths degrees of freedom can be written as a function of the initial conditions and of the operator $x(t)$ as $[30,42,66]$

$$
\begin{aligned}
X_{k, v}(t)= & X_{k, v}\left(t_{0}\right) \cos \left[\omega_{k, v}\left(t-t_{0}\right)\right] \\
& +\frac{P_{k, v}\left(t_{0}\right)}{m_{k, v} \omega_{k, v}} \sin \left[\omega_{k, v}\left(t-t_{0}\right)\right] \\
& +\frac{c_{k, v}}{m_{k, v} \omega_{k, v}} \int_{t_{0}}^{t} d s g_{v}(s) x(s) \sin \left[\omega_{k, v}(t-s)\right] .
\end{aligned}
$$

Substituting these expressions into Eqs. (7) one obtains the generalized quantum Langevin equation for the oscillator

$$
\begin{aligned}
\ddot{x}(t) & +\omega_{0}^{2} x(t)+\int_{t_{0}}^{+\infty} d s \sum_{\nu=1}^{N} g_{v}(t) \gamma_{v}(t-s) \\
& \times\left[\dot{g}_{\nu}(s) x(s)+\dot{x}(s) g_{v}(s)\right] \\
& =\frac{1}{m} \sum_{\nu=1}^{N} g_{v}(t) \xi_{v}(t) .
\end{aligned}
$$

Here,

$$
\gamma_{\nu}(t)=\frac{\theta(t)}{m} \sum_{k=1}^{\infty} \frac{c_{k, \nu}^{2}}{m_{k, \nu} \omega_{k, \nu}^{2}} \cos \left(\omega_{k, \nu} t\right)
$$

represents the memory damping kernel, which is assumed in a large universality class such that it vanishes at long times $\left[\lim _{t \rightarrow \infty} \gamma_{\nu}(t) \rightarrow 0\right]$. Here, $\theta(t)$ the Heaviside step function. Note that on the right-hand side of Eq. (10) we have dropped the inhomogeneous term $-x\left(t_{0}\right) \sum_{v=1}^{N} g_{v}(t) g_{v}\left(t_{0}\right) \gamma_{v}\left(t-t_{0}\right)$ since it is a typical transient contribution that decays to zero at $t>0$ times (we assume the initial condition at time $t_{0} \rightarrow-\infty$ ). In addition, we recall that the time-dependent couplings $g_{v}(t)$ are switched on at $t=t_{0}^{+}$. The operator

$$
\begin{aligned}
\xi_{v}(t)=\sum_{k=1}^{\infty} c_{k, v}[ & X_{k, v}\left(t_{0}\right) \cos \omega_{k, v}\left(t-t_{0}\right) \\
& \left.+\frac{P_{k, v}\left(t_{0}\right)}{m_{k, v} \omega_{k, v}} \sin \omega_{k, v}\left(t-t_{0}\right)\right]
\end{aligned}
$$

is the fluctuating force of the bath $v$, and it depends explicitly on the initial conditions of the bath position and momentum operators $X_{k, v}\left(t_{0}\right)$ and $P_{k, v}\left(t_{0}\right)$. It has zero average $\left\langle\xi_{v}(t)\right\rangle=0$, as one can see using the initial thermal conditions (6). Note that here and below we denote the quantum average of any operator $O$ as $\langle O\rangle=\operatorname{Tr}\left[O \rho\left(t_{0}\right)\right]$.

The corresponding correlation functions $\left\langle\xi_{v}(t) \xi_{v^{\prime}}\left(t^{\prime}\right)\right\rangle$ are evaluated by expressing them in terms of the bath 
spectral density defined as [51]

$$
\mathcal{J}_{\nu}(\omega)=\frac{\pi}{2} \sum_{k=1}^{\infty} \frac{c_{k, v}^{2}}{m_{k, v} \omega_{k, v}} \delta\left(\omega-\omega_{k, v}\right)
$$

We have

$$
\left\langle\xi_{v}(t) \xi_{v^{\prime}}\left(t^{\prime}\right)\right\rangle=\delta_{v, v^{\prime}}\left[\mathcal{L}_{v}^{(+)}\left(t-t^{\prime}\right)-i \mathcal{L}_{v}^{(-)}\left(t-t^{\prime}\right)\right],
$$

where

$$
\begin{aligned}
& \mathcal{L}_{v}^{(+)}(t)=\int_{0}^{\infty} \frac{d \omega}{\pi} \mathcal{J}_{v}(\omega) \operatorname{coth}\left(\frac{\omega}{2 T_{v}}\right) \cos (\omega t), \\
& \mathcal{L}_{v}^{(-)}(t)=\int_{0}^{\infty} \frac{d \omega}{\pi} \mathcal{J}_{v}(\omega) \sin (\omega t)
\end{aligned}
$$

describe the symmetric and antisymmetric contributions, respectively. Similarly, the damping kernel $\gamma_{v}(t)$ in Eq. (11) can be written as

$$
\gamma_{\nu}(t)=\frac{2}{\pi m} \theta(t) \int_{0}^{\infty} d \omega \frac{\mathcal{J}_{\nu}(\omega)}{\omega} \cos (\omega t) .
$$

\section{B. Average thermodynamic quantities}

We now focus on the long time behavior of the full system (QHO plus baths). In this limit, due to the presence of dissipation, the transient dynamics is washed out and the total system reaches a periodic state sustained by the coupling drives. The key thermodynamic quantities to be inspected are then the driving powers and the heat currents averaged over the period $\mathcal{T}$ of the cycle. To obtain these quantities, we start by defining their time evolutions $P_{v}(t)$ and $J_{v}(t)$. The injected power $P_{v}(t)$, associated with the temporal variation of the system-bath couplings $g_{v}(t)$, is defined as the quantum average [30,37]

$$
P_{v}(t)=\operatorname{Tr}\left[\frac{\partial H_{\mathrm{int}, v}^{(t)}}{\partial t} \rho(t)\right]
$$

with $\rho(t)$ the total density matrix at time $t$. The corresponding mean power $P_{v}$, averaged over the cycle, is then given by

$$
P_{\nu}=\frac{1}{\mathcal{T}} \int_{0}^{\mathcal{T}} d t P_{v}(t)
$$

with the total average power $P=\sum_{v=1}^{N} P_{v}$.

The time-dependent heat current, associated with the bath $v$, is given by the change in time of the reservoir energy $[61,71,72]$. It reads

$$
J_{v}(t)=-\operatorname{Tr}\left[H_{v} \dot{\rho}(t)\right],
$$

where the minus sign implies a positive $J_{v}(t)$ when the energy flows from the reservoir into the quantum system.
The mean heat current, averaged over the cycle, is then

$$
J_{v}=\frac{1}{\mathcal{T}} \int_{0}^{\mathcal{T}} d t J_{v}(t)
$$

Note that, in the considered periodic regime, the total power injected from the coupling drives is balanced by the reservoir heat currents and it fulfills the relation

$$
P+\sum_{\nu=1}^{N} J_{v}=0
$$

This equality is derived using the fact that both $\operatorname{Tr}\left[H_{S} \rho(t)\right]$ and $\operatorname{Tr}\left[H_{\text {int, }}^{(t)} \rho(t)\right]$ show a periodic behavior at long times [61]. Equation (21) can be interpreted as the analogue of the first law of thermodynamics. To better see this point, we can rewrite it in a more standard form by introducing the total mean work per cycle $W$ and the mean heat $Q_{v}$ of the $v$ bath $[30,37,61]$. These two quantities are directly given by $W=P \mathcal{T}$ and $Q_{v}=J_{v} \mathcal{T}$ and fulfill the relation $W+\sum_{v=1}^{N} Q_{v}=0$.

Another key quantity to consider is the time average entropy production rate that is linked to the currents through the relation (see, e.g., Ref. [9])

$$
\dot{S}=-\sum_{\nu=1}^{N} \frac{J_{v}}{T_{v}} .
$$

Note that, from the second law of thermodynamics, we always have $\dot{S} \geq 0$, which in particular implies, for isothermal baths $\left(T_{v}=T\right)$ and using Eq. (21), a positive total power $P=T \dot{S} \geq 0$. This relation is consistent with the Kelvin-Planck statement of the second law: a cyclic transformation whose sole effect is to convert heat, extracted from a source at constant temperature, into work is impossible $[73,74]$.

We conclude this general part by writing the explicit expressions of the time-dependent powers (17) and heat currents (19) in terms of the quantum average over the bath and system variables. In particular, for the time-dependent heat currents, inserting the explicit expressions of the Hamiltonian contributions into Eqs. (2)-(3) one arrives at

$$
J_{v}(t)=-g_{v}(t) \sum_{k=1}^{\infty} \frac{c_{k, v}}{2 m_{k, v}}\left\langle\left[x(t) P_{k, v}(t)+P_{k, v}(t) x(t)\right]\right\rangle \text {. }
$$

Now, using the solution of the EOM (8) and (9), we have

$$
\begin{aligned}
J_{v}(t)= & -\frac{g_{v}(t)}{2}\left\langle x(t) \dot{\xi}_{v}(t)+\dot{\xi}_{v}(t) x(t)\right\rangle \\
& -g_{v}(t) \int_{-\infty}^{t} d s\langle x(t) x(s)+x(s) x(t)\rangle g_{v}(s) \\
& \times \frac{d}{d t} \mathcal{L}_{v}^{(-)}(t-s) .
\end{aligned}
$$


Following the same steps, the time-dependent power contributions read

$$
\begin{aligned}
P_{v}(t)= & -\dot{g}_{v}(t)\left\langle x(t) \xi_{v}(t)\right\rangle \\
& +m \dot{g}_{v}(t) \int_{-\infty}^{+\infty} d s \gamma_{v}(t-s) \frac{d}{d s}\left[g_{v}(s)\langle x(t) x(s)\rangle\right] .
\end{aligned}
$$

These expressions represent the key starting point in order to evaluate all thermodynamic quantities after averaging over the period of the cycle.

\section{GENERAL APPROACH: OUT-OF-EQUILIBRIUM GREEN'S FUNCTION}

To evaluate the above thermodynamic quantities, it is first necessary to solve the EOM of the oscillator position operator $x(t)$ in Eq. (10) $[42,43,66,75]$. To this end, we introduce the associated retarded Green function $G\left(t, t^{\prime}\right)$ that fulfills the equation

$$
\begin{aligned}
& \ddot{G}\left(t, t^{\prime}\right)+\omega_{0}^{2} G\left(t, t^{\prime}\right)+\int_{-\infty}^{+\infty} d s \sum_{v=1}^{N} g_{v}(t) \gamma_{v}(t-s) \\
& \quad \times\left[\dot{g}_{v}(s) G\left(s, t^{\prime}\right)+g_{v}(s) \dot{G}\left(s, t^{\prime}\right)\right] \\
& \quad=\delta\left(t-t^{\prime}\right)
\end{aligned}
$$

with $G\left(t, t^{\prime}\right)=0$ for $t \leq t^{\prime}$. Here, the dot denotes the derivative with respect to the first argument. Note that, due to the breaking of temporal translation, caused by the timedependent couplings, the Green function depends separately on $t, t^{\prime}$ and not on their difference $t-t^{\prime}$ only. As already mentioned, we are interested in finding solutions in the long time limit, where the memory of the initial state is lost and the system reaches a periodic state sustained by the drives. In this regime, the time evolution of the position operator $x(t)$ can be expressed directly as a time integral of the retarded Green function with the inhomogeneous term:

$$
x(t)=\sum_{v=1}^{N} \int_{-\infty}^{+\infty} d t^{\prime} G\left(t, t^{\prime}\right) \frac{1}{m} g_{v}\left(t^{\prime}\right) \xi_{v}\left(t^{\prime}\right)
$$

This important relation allows us to express all quantum correlation averages, which define the driving powers and the heat currents, in terms of the resolvent Green function $G\left(t, t^{\prime}\right)$.

In addition, in the long time limit, the Green function acquires a peculiar time property $[42,43,75-78]$. Indeed, even if $G\left(t, t^{\prime}\right)$ is not periodic, the Fourier transform

$$
\tilde{G}(t, \omega)=\int_{-\infty}^{+\infty} d t^{\prime} e^{i \omega\left(t-t^{\prime}\right)} G\left(t, t^{\prime}\right)
$$

obeys $\tilde{G}(t+\mathcal{T}, \omega)=\tilde{G}(t, \omega)$ and it can be written in terms of the Fourier series:

$$
\tilde{G}(t, \omega)=\sum_{m=-\infty}^{+\infty} \tilde{G}_{m}(\omega) e^{-i m \Omega t}
$$

with $\tilde{G}_{m}(\omega)$ the so-called Floquet coefficients. Note that, for a static drive, only the $m=0$ component would be present in the series expansion. Using the above relation, we can write a rather compact expression for $G\left(t, t^{\prime}\right)$ :

$$
G\left(t, t^{\prime}\right)=\sum_{m=-\infty}^{+\infty} \int_{-\infty}^{+\infty} \frac{d \omega}{2 \pi} e^{-i \omega\left(t-t^{\prime}\right)} \tilde{G}_{m}(\omega) e^{-i m \Omega t} .
$$

Inserting Eq. (30) into Eq. (26) one obtains the following algebraic set of equations for the Floquet coefficients (see Appendix A for more details):

$$
\begin{aligned}
\tilde{G}_{m}(\omega)= & \chi_{0}(\omega) \delta_{m, 0}-\chi_{0}(\omega+m \Omega) \\
& \times \sum_{n \neq 0} \tilde{k}_{n}[\omega+(m-n) \Omega] \tilde{G}_{m-n}(\omega) .
\end{aligned}
$$

Here

$$
\tilde{k}_{n}(\omega)=-i \sum_{\nu=1}^{N} \sum_{m=-\infty}^{+\infty} g_{m, \nu} g_{n-m, \nu}(\omega+m \Omega) \tilde{\gamma}_{\nu}(\omega+m \Omega)
$$

represents the influence kernel of the driving due to the baths, with $\widetilde{\gamma}_{v}(\omega)$ the Fourier transform of $\gamma_{v}(t)$ in Eq. (16). In Eq. (31), we have also introduced the "static" retarded Green function in the spectral domain

$$
\chi_{0}(\omega)=-\frac{1}{\omega^{2}-\omega_{0}^{2}-\tilde{k}_{0}(\omega)},
$$

which contains the $n=0$ component, $\tilde{k}_{0}(\omega)$, of the bath kernel $\tilde{k}_{n}(\omega)$.

The important point of the present approach is that the knowledge of the Floquet coefficients $\tilde{G}_{m}(\omega)$ allows us to solve the full dynamics of the system at long times. We underline that the solution of the coupled equations (31) in general should be tackled by means of numerical techniques, i.e., by exploiting exact diagonalization and inversion of large matrices or using an iterative procedure.

We conclude this general part by deriving the expressions for the average heat currents and powers written in terms of the Floquet coefficients. Here, we quote the main steps, presenting details in Appendix B. First, we consider the expression for the position operator in Eq. (27) and we insert it into the heat current $J_{v}(t)$ in Eq. (24). The result is 


$$
\begin{aligned}
J_{v}(t)= & -\frac{g_{v}(t)}{m} \int_{-\infty}^{+\infty} d t^{\prime} G\left(t, t^{\prime}\right) g_{v}\left(t^{\prime}\right) \dot{\mathcal{L}}_{v}^{(+)}\left(t-t^{\prime}\right)-\frac{2 g_{v}(t)}{m^{2}} \int_{-\infty}^{t} d s \int_{-\infty}^{+\infty} d t_{1} \int_{-\infty}^{+\infty} d t_{2} g_{v}(s) \dot{\mathcal{L}}_{v}^{(-)}(t-s) \\
& \times G\left(t, t_{1}\right) G\left(s, t_{2}\right) \sum_{v_{1}=1}^{N} g_{v_{1}}\left(t_{1}\right) g_{v_{1}}\left(t_{2}\right) \mathcal{L}_{v_{1}}^{(+)}\left(t_{1}-t_{2}\right),
\end{aligned}
$$

where, as before, the dot denotes the derivative with respect to the first argument. Using now the Fourier integrals and series of Eqs. (4), (30), we obtain the heat current after the time average over the cycle:

$$
\begin{aligned}
J_{v}= & \sum_{n_{1}, n_{2}=-\infty}^{+\infty} g_{n_{1}, v} g_{n_{2}, v} \int_{-\infty}^{+\infty} \frac{d \omega}{2 \pi m}\left[-i \mathcal{J}_{\nu}(\omega) \omega \operatorname{coth}\left(\frac{\omega}{2 T_{v}}\right) \tilde{G}_{-\left(n_{1}+n_{2}\right)}\left(\omega+n_{2} \Omega\right)\right. \\
& -\sum_{\nu_{1}=1}^{N} \sum_{m_{1}=-\infty}^{+\infty} \sum_{n_{3}, n_{4}=-\infty}^{+\infty} g_{n_{3}, \nu_{1}} g_{n_{4}, \nu_{1}} \frac{\mathcal{J}_{\nu_{1}}(\omega)}{m} \operatorname{coth}\left(\frac{\omega}{2 T_{v_{1}}}\right)\left[\omega-\Omega\left(n_{2}+n_{4}+m_{1}\right)\right] \mathcal{J}_{v}\left[\omega-\Omega\left(n_{2}+n_{4}+m_{1}\right)\right] \\
& \left.\times \tilde{G}_{m_{1}}\left(-\omega+n_{4} \Omega\right) \tilde{G}_{-\left(n_{\text {tot }}+m_{1}\right)}\left(\omega+n_{3} \Omega\right)\right]
\end{aligned}
$$

with $n_{\text {tot }}=n_{1}+n_{2}+n_{3}+n_{4}$. In the above expression the spectral density $\mathcal{J}_{v}(\omega)$ is extended at negative frequencies by requiring the odd property $\mathcal{J}_{v}(\omega)=-\mathcal{J}_{v}(-\omega)$.

Starting from Eq. (25) and following similar steps we also obtain the average power associated with the $v$ th bath [see Eq. (B15) in Appendix B].

\section{RESULTS AND DISCUSSION}

The formalism developed so far is general and allows one to exactly evaluate all stationary heat currents and power contributions in a multiterminal configuration with $v$ reservoirs in the presence of time-dependent drives modulating the various system-bath couplings.

Hereafter, we consider ohmic baths that describe a wide class of dissipative environments, for instance metallic fermionic leads [51] or superconducting circuit elements $[67,68,70]$, with spectral densities

$$
\mathcal{J}_{v}(\omega)=m \gamma_{v} \omega e^{-|\omega| / \omega_{c}},
$$

where $\gamma_{v}$ quantifies the interaction strength between the $v$ th bath and the system, and $\omega_{c}$ is the bath cutoff frequency kept as the largest energy scale. This important class of dissipation has damping kernels $\gamma_{\nu}(t)$ local in time, $\gamma_{v}(t)=2 \gamma_{v} \theta(t) \delta(t)$, with Fourier transform

$$
\tilde{\gamma}_{\nu}(\omega)=\gamma_{\nu}
$$

In this case, the dissipative kernel $\tilde{k}_{n}(\omega)$ in Eq. (32) can be rewritten as the time average

$$
\tilde{k}_{n}(\omega)=\frac{1}{\mathcal{T}} \int_{0}^{\mathcal{T}} d t e^{i n \Omega t} \sum_{\nu=1}^{N} \gamma_{\nu}\left[g_{v}(t) \dot{g}_{\nu}(t)-i \omega g_{v}^{2}(t)\right] .
$$

It is worth stressing that, although the damping kernels $\gamma_{v}(t)$ are local in time with ohmic spectral functions, this does not imply a Markovian dynamics. Indeed, the noise terms in Eq. (12) retain memory and thus non-Markovian signatures. It is only in the classic regime, $T \gg \omega_{0}$, that noise terms loose memory, as one can see looking at the correlators (14) that become

$$
\left\langle\xi_{v}(t) \xi_{v^{\prime}}\left(t^{\prime}\right)\right\rangle \approx 2 m \gamma_{v} T_{v} \delta_{v, v^{\prime}} \delta\left(t-t^{\prime}\right) .
$$

\section{A. Ratchet-induced cooling and refrigeration}

We now discuss the average heat flows induced by the temporal modulation of the system-bath couplings. The aim is to find particular regimes where time-dependent coupling acts in a thermodynamically efficient way, opposite to the usually expected purely dissipative regime.

To this end, we consider peculiar shapes of the drives $g_{\nu}(t)$ that break time-translational invariance and can allow for the so-called heat ratchet effect. It has been shown that asymmetric drives, by relying on dynamical symmetry breaking, are indeed able to produce, e.g., directed heat flow and heat rectification $[63,64,79]$. Dynamical breaking of temporal reflection symmetry, and thus the ratchet effect, can be induced by nonlinear harmonic mixing of different frequencies of the drives. Note that such mixing is possible, in spite of the fact that we are considering 
a harmonic oscillator system, due to the periodic drive. Importantly, and differently from previous studies, in our case the ratchet effect is achieved by suitably engineering the modulation of the system-bath couplings only. To proceed further, we note that the expression in Eq. (38) is quite illuminating, since it allows us to find a particular class of time-dependent couplings $g_{v}(t)$, which verify $\sum_{\nu=1}^{N} \gamma_{\nu} \dot{g}_{\nu}(t) g_{v}(t)=0$. This implies the constraint

$$
\sum_{\nu=1}^{N} \gamma_{\nu} g_{v}^{2}(t)=\gamma
$$

with $\gamma$ an effective damping that feels both dissipation amplitudes $\gamma_{v}$ and the associated harmonic components. Notably, the above constraint implies that only the static contribution $\tilde{k}_{0}(\omega)$ in Eq. (38) is different from zero, with

$$
\tilde{k}_{n}(\omega)=-i \omega \gamma \delta_{n, 0}
$$

The above relation allows an exact solution of the set of coupled algebraic equations in Eq. (31). Indeed, one finds that

$$
\tilde{G}_{m}(\omega)=\chi_{0}(\omega) \delta_{m, 0}
$$

with only the static retarded Green function component, even if the couplings $g_{v}(t)$ are still time dependent. The resulting expressions for the average heat currents are now simplified by substituting Eq. (42) into Eq. (35). We have

$$
\begin{aligned}
J_{\nu}= & \gamma_{\nu} \int_{-\infty}^{+\infty} \frac{d \omega}{2 \pi}\left\{\omega^{2} \operatorname{coth}\left(\frac{\omega}{2 T_{\nu}}\right) e^{-|\omega| / \omega_{c}} \sum_{n_{1}=-\infty}^{+\infty}\left|g_{n_{1}, v}\right|^{2} \operatorname{Im} \chi_{0}\left(\omega-n_{1} \Omega\right)-\sum_{\nu_{1}=1}^{N} \sum_{n_{1,3,4}=-\infty}^{+\infty} g_{n_{1}, \nu} g_{-\left(n_{1}+n_{3}+n_{4}\right), \nu}\right. \\
& \left.\times g_{n_{3}, \nu_{1}} g_{n_{4}, \nu_{1}} \gamma_{\nu_{1}} \omega \operatorname{coth}\left(\frac{\omega}{2 T_{\nu_{1}}}\right)\left[\omega+\Omega\left(n_{1}+n_{3}\right)\right]^{2} e^{-|\omega| / \omega_{c}} \chi_{0}\left(-\omega+n_{4} \Omega\right) \chi_{0}\left(\omega+n_{3} \Omega\right) e^{-\left|\omega+\Omega\left(n_{1}+n_{3}\right)\right| / \omega_{c}}\right\} .
\end{aligned}
$$

Several choices of $g_{v}(t)$ can fulfill constraint (40), and we believe that this is a convenient setting to grasp the physics that can be induced by time-dependent system-bath couplings. To proceed further, we now focus on the simplest configuration that consists of two reservoirs $v=1,2$ only. Despite its simplicity, it represents the prototypical model in order to investigate the role of driven system-bath couplings on thermodynamic performances. As we will show, this two terminal configuration is already sufficient to perform nontrivial thermodynamic tasks.

First, we note that monochromatic drives such as $g_{1}(t)=\sqrt{\gamma / \gamma_{1}} \cos (\Omega t)$ and $g_{2}(t)=\sqrt{\gamma / \gamma_{2}} \sin (\Omega t)$ satisfy constraint (40), but they will not produce any harmonic mixing, thus resulting in a rather trivial dynamics. For instance, in such a case, for isothermal baths, the average heat currents are always dissipated into the reservoirs, i.e., both $J_{1}$ and $J_{2}$ have negative signs, independently of the operating regime (driving frequency or temperature range). Therefore, in order to observe nontrivial effects, we choose

$$
\begin{aligned}
& g_{1}(t)=\cos (\Omega t), \\
& g_{2}(t)=\sqrt{\frac{\gamma}{\gamma_{2}}} \sqrt{1-\kappa \cos ^{2}(\Omega t)},
\end{aligned}
$$

where we have defined the effective asymmetry $0<\kappa=$ $\gamma_{1} / \gamma<1$. This choice allows harmonic mixing between the two drives, as one can see by using Eq. (4) and evaluating the associated Fourier coefficients. Indeed, one obtains

$$
\begin{aligned}
& g_{n, 1}=\frac{1}{2}\left[\delta_{n, 1}+\delta_{n,-1}\right], \\
& g_{n, 2}=\sqrt{\frac{\gamma}{\gamma_{2}}}\left[\frac{2}{\pi} E(\kappa) \delta_{n, 0}-\frac{\kappa}{8}{ }_{2} F_{1}\left(\frac{1}{2}, \frac{3}{2}, 3 ; \kappa\right) \delta_{n, \pm 2}-\frac{(n-3) ! !}{(n / 2) ! 2^{3 n / 2}} \kappa^{n / 2}{ }_{2} F_{1}\left(\frac{n-1}{2}, \frac{n+1}{2}, n+1 ; \kappa\right) \delta_{n, \pm 2 p}\right],
\end{aligned}
$$

where $E(x),{ }_{2} F_{1}(a, b, c ; x)$ are the elliptic and hypergeometric functions, respectively, and $p$ represents any integer $p \geq 2$, indicating higher-harmonic contributions. It is thus manifest that $g_{1}(t)$ contains odd harmonics, while $g_{2}(t)$ has even components only; therefore, looking at Eq. (43), an interplay between odd and even components is expected. 

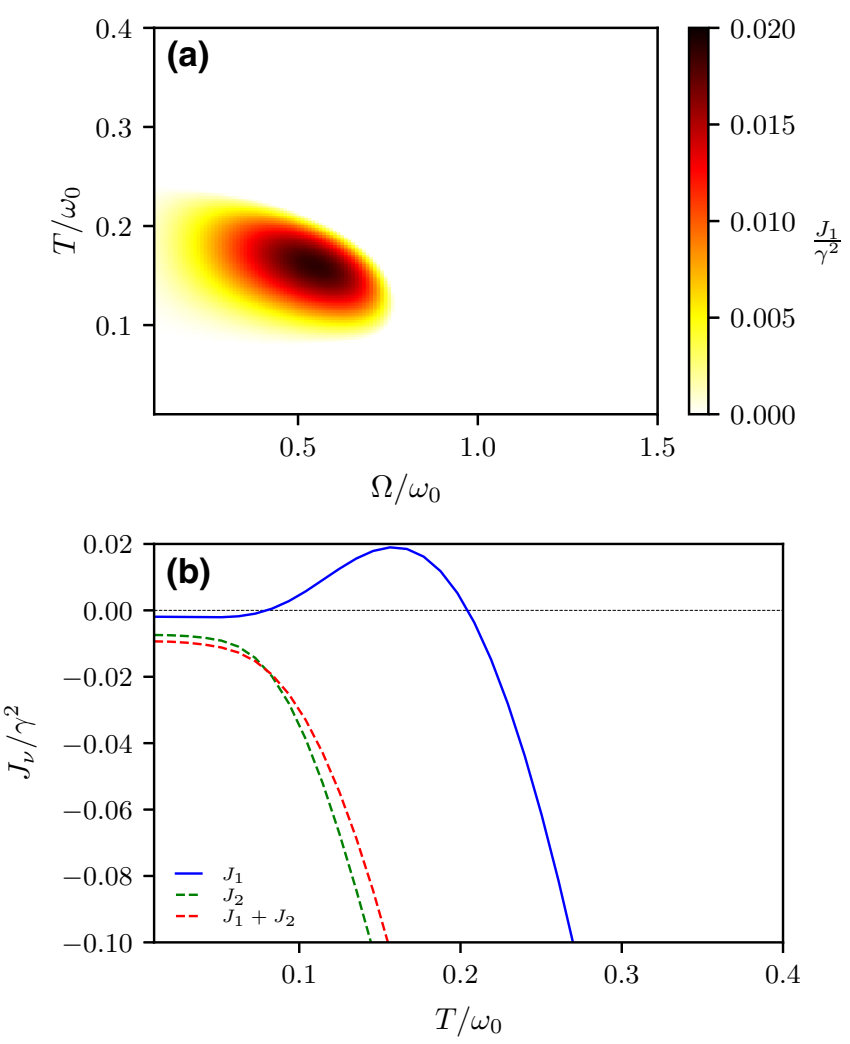

FIG. 2. Average heat currents with asymmetric coupling $\kappa=$ $\gamma_{1} / \gamma=0.2$ and isothermal baths $T_{1}=T_{2}=T$. (a) Density plot of the average heat current $J_{1} / \gamma^{2}$ as a function of driving frequency $\Omega / \omega_{0}$ and temperature $T / \omega_{0}$. Colored regions refer to positive values of $J_{1} / \gamma^{2}$ (heat flow from the bath $v=1$ toward the system). (b) Average heat currents $J_{1} / \gamma^{2}, J_{2} / \gamma^{2}$, and $\left(J_{1}+\right.$ $\left.J_{2}\right) / \gamma^{2}$ as a function of temperature $T / \omega_{0}$ at frequency $\Omega=$ $0.55 \omega_{0}$. Damping strength is $\gamma=0.01 \omega_{0}$ and $\omega_{c}=500 \omega_{0}$.

We have evaluated the average heat currents $J_{v}$ by means of numerical integration of Eq. (43), using Eq. (45) for different values of $\kappa$.

An illuminating example is shown in Fig. 2 where we consider isothermal baths and a representative value for the effective damping strength and asymmetry. Here, finite heat currents are obtained akin to a dynamical Peltier contribution. In Fig. 2(a) we show a density plot of the average heat current $J_{1}$ versus frequency $\Omega / \omega_{0}$ and temperature $T / \omega_{0}$. Note that, for the specific choice of the drives in Eqs. (44), the $v=1$ reservoir is the one that may exhibit nontrivial phenomena. This can be seen from the density plot, where positive values $J_{1}>0$ appear (see the colored area). This means the presence of an induced ratchet cooling phenomenon, with a heat current that flows from the $v=1$ reservoir toward the system. This behavior is counterintuitive, since in the absence of additional external driving forces acting on the system, one would naively expect a dissipative heat current induced by the modulation of the system-bath couplings, flowing from the system to the reservoir.

It is important to underline that these spots of positive $J_{1}$ are present only in the deep quantum regime $T \ll \omega_{0}$ where non-Markovian contributions are present. Indeed, in the classical regime, where the whole system looses memory (Markovian dynamics) [see Eq. (39)], both average heat currents, resulting from dynamical Peltier contribution, always have a negative sign (see Appendix $\mathrm{C}$ for a rigorous proof of this result).

In Fig. 2(b), a cut of the density plot at fixed driving frequency $\Omega$ is shown. Since the region with positive $J_{1}$ is always found at frequencies $\Omega \leq \omega_{0}$, we choose $\Omega=$ $0.55 \omega_{0}$ here. In addition to $J_{1}$, we plot both the average heat current $J_{2}$, flowing in the other reservoir $v=2$, and the sum of the two heat currents $J_{1}+J_{2}$. We underline that the two latter quantities are always negative in the explored parameter regions. This confirms a total power $P=-\left(J_{1}+J_{2}\right)$, supplied by the external coupling drives, always positive, in agreement with the relation $P=T \dot{S} \geq$ 0 [see Eq. (22)].

Importantly, Fig. 2 shows that a nontrivial cooling mechanism can emerge, with $J_{1}>0$, without requiring any external forces directly coupled to the quantum system. To better investigate this phenomenon induced by time-dependent drives acting on the system-bath couplings, we have studied the behavior of $J_{1}$ for different values of the asymmetry parameter $\kappa$. Indeed by varying $\kappa$, one can change the asymmetry between the couplings and, at the same time, increase or decrease the mixing of different harmonics of the time-dependent signals. As a general result, we observe qualitatively similar behaviors as those presented in Fig. 2. Common ingredients to obtain the cooling phenomenon are: a ratchet dynamics, a non-Markovian behavior, present only in the deep quantum regime $T \ll \omega_{0}$, and driving frequencies $\Omega<\omega_{0}$. Concerning the last inequality, we can say that the stronger the asymmetry (smaller values of $\kappa$ ), the closer the frequency to resonance $\left(\Omega \rightarrow \omega_{0}^{-}\right)$in the region with positive $J_{1}>0$.

In Fig. 3(a), we plot the maximum positive value of $J_{1} / \gamma^{2}$ as a function of the asymmetry parameter $\kappa$. Here, a nonmonotonic behavior is visible, starting linearly at $\kappa \rightarrow 0$ with a maximum around $\kappa \sim 0.2$. In Fig. 3(b), the role of $\kappa$ is inspected by means of a figure of merit defined as the ratio between the maximum value of the average heat current $\operatorname{Max}\left[J_{1}\right]$ and the corresponding total amount of power $P$ supplied by the drives. In this case, a decreasing behavior is present and it clearly emerges that $\kappa \ll 1$ is the optimal choice: the normalized cooling effect is higher with stronger asymmetry and it tends to saturate for sufficiently low values of $\kappa$. Note that a similar, monotonic and decreasing, behavior is also found if one considers the maximum of the ratio $\operatorname{Max}\left[J_{1} / P\right]$ as a function of the $\kappa$, as depicted in the inset of Fig. 3(b). 

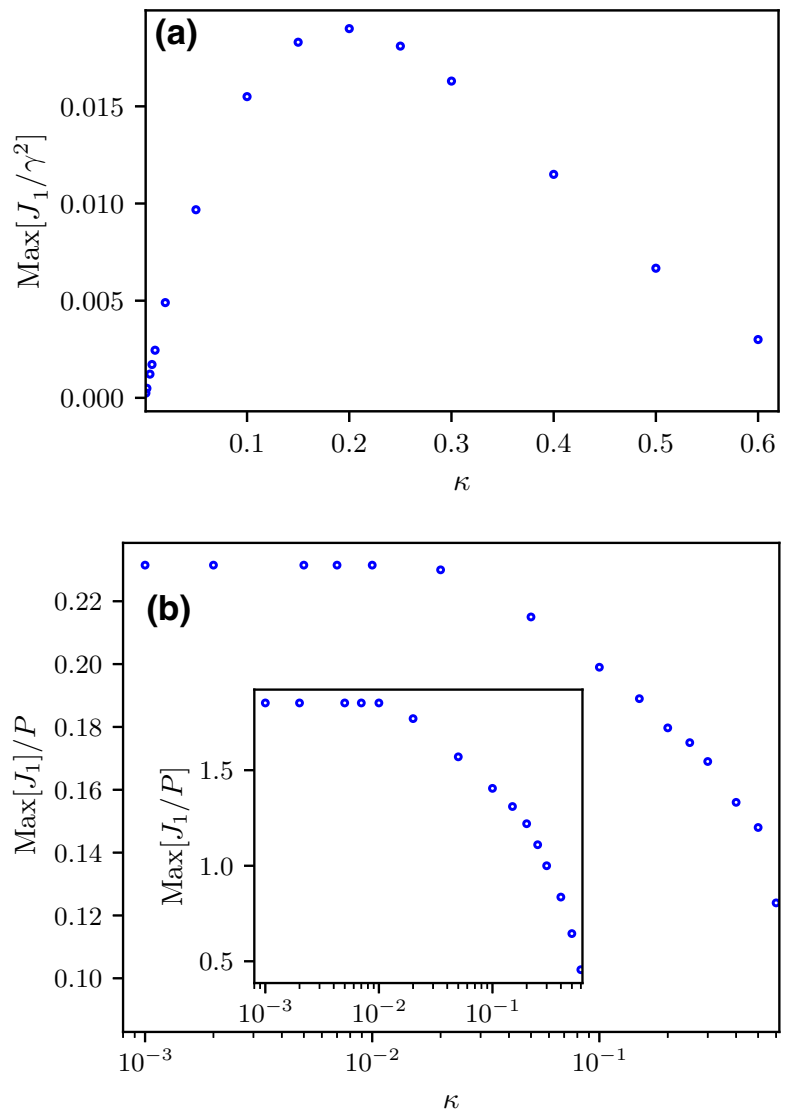

FIG. 3. Asymmetry dependence of cooling. (a) Maximum positive value of $J_{1} / \gamma^{2}$, achieved in the $(\Omega, T)$ plane, as a function of the asymmetry parameter $\kappa$. (b) Ratio between the maximum positive value of $J_{1}$ and the corresponding average total power $P$ supplied by the drives as a function of $\kappa$. The inset also shows the monotonic behavior of the maximum of the ratio $\operatorname{Max}\left[J_{1} / P\right]$ as a function of $\kappa$. Other parameters are as in Fig. 2(a).

Below, we therefore focus on the strongly asymmetric case $\kappa \ll 1$. Here, a perturbative expansion can be carried out by approximating $g_{v}(t)$ in Eqs. (44) as

$$
\begin{aligned}
& g_{1}(t)=\cos (\Omega t), \\
& g_{2}(t) \approx \sqrt{\frac{\gamma}{\gamma_{2}}}\left[1-\frac{\kappa}{2} \cos ^{2}(\Omega t)\right] .
\end{aligned}
$$

It is worth noting that, within this perturbative expansion, up to linear order in $\kappa$, the constraint in Eq. (40) is no more guaranteed and one should carefully check that Eq. (42) is still satisfied at the appropriate truncation of the expansion. This is indeed the case: we have verified (see Appendix D) that all other contributions start at order $O\left(\kappa^{2}\right)$. Consistently with such a perturbative approach, the corresponding average heat currents are evaluated up to linear order in $\kappa$.
The average heat current, associated with the $v=1$ reservoir, is (see Appendix D for details)

$$
\begin{aligned}
J_{1}= & \kappa \gamma \int_{-\infty}^{+\infty} \frac{d \omega}{4 \pi}\left\{-\left(\omega^{2}+\Omega^{2}\right) \operatorname{Im} \chi_{0}(\omega) \operatorname{coth}\left(\frac{\omega}{2 T_{2}}\right)\right. \\
& \left.+\omega^{2} \operatorname{Im} \chi_{0}(\omega+\Omega) \operatorname{coth}\left(\frac{\omega}{2 T_{1}}\right)\right\} .
\end{aligned}
$$

Similarly, the one for reservoir $v=2$ reads

$$
\begin{aligned}
J_{2}= & \kappa \gamma \int_{-\infty}^{+\infty} \frac{d \omega}{4 \pi}\left\{\omega^{2} \operatorname{Im} \chi_{0}(\omega) \operatorname{coth}\left(\frac{\omega}{2 T_{2}}\right)\right. \\
& \left.-\omega(\omega+\Omega) \operatorname{Im} \chi_{0}(\omega+\Omega) \operatorname{coth}\left(\frac{\omega}{2 T_{1}}\right)\right\} .
\end{aligned}
$$

These expressions are well behaved and therefore we have safely taken the $\omega_{c} \rightarrow \infty$ limit for the cutoff of the ohmic spectral functions. Finally, the average total power is obtained from $P=-\left(J_{1}+J_{2}\right)$.

The above expressions can be analytically evaluated in closed form by resorting to proper Matsubara resummation and integration in the complex plane. Details and full expressions can be found in Appendix D.

We now discuss the cooling properties by inspecting the behavior of the average heat currents in Eqs. (47)-(48). We start by pointing out that the possibility to achieve regions with positive $J_{1}$ also depends on the value of the effective damping $\gamma$. Indeed, if $\gamma$ is too strong, all heat currents $J_{v}$ dissipate into the reservoirs, with $J_{v}<0$. To elucidate this point, in Fig. 4(a) we have depicted the maximum positive value of $J_{1}$, achieved in the $(\Omega, T)$ plane, as a function of the effective damping $\gamma$ using Eq. (47) valid for strong asymmetry (in the figure, $\kappa=0.01$ ). Here, for $\gamma \rightarrow 0$, we have $J_{1} \propto \gamma$, while increasing $\gamma$ above a critical value $\gamma_{c}$, $J_{1}$ becomes negative in the whole parameter range. For the specific value of $\kappa=0.01$, we obtain $\gamma_{c}=0.285 \omega_{0}$. A qualitatively similar behavior is also found for other values of $\kappa$, outside the perturbative regime, only with small changes in the precise value of $\gamma_{c}$.

It is important to stress that, even if there are regions at fixed temperature with $J_{1}>0$, decreasing the temperature toward $T \rightarrow 0$, the average heat current $J_{1}$ always becomes negative, consistent with Nernst's unattainability principle $[42,43]$. To be more quantitative, we look in the above perturbative regime for the zeroes of $J_{1}(\Omega, T)$ at a given effective damping strength $\gamma$. These are points in the $(\Omega, T)$ plane. We define the minimum achievable cooling temperature $T_{\min }$ as that associated with the zero point with the minimum temperature among all the possible zeros of $J_{1}$. By varying the effective damping $\gamma$ we reproduce the function $T_{\min }(\gamma)$ shown in Fig. 3(b). As we can see, the weaker the damping (and consequently the cooling power), the lower the cooling temperature $T_{\min }$, so that 

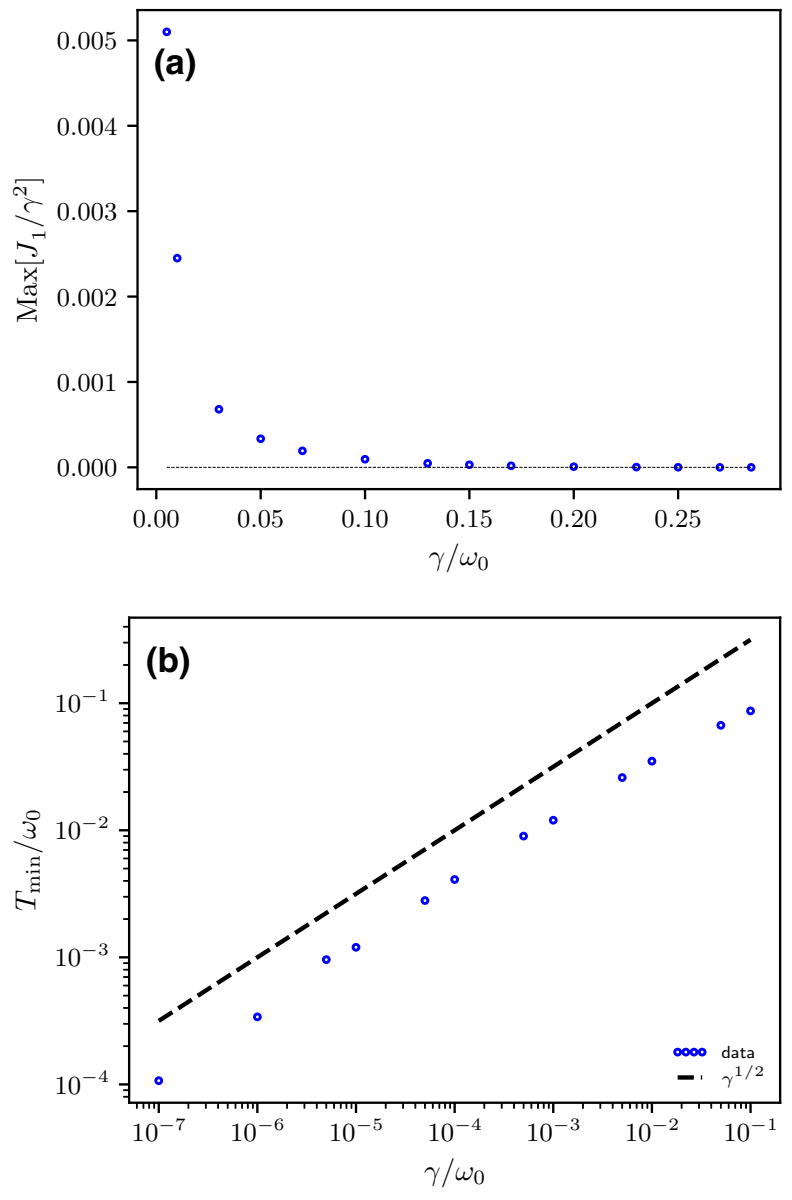

FIG. 4. Cooling power and minimum achievable temperature versus effective damping. (a) Maximum positive value of $J_{1} / \gamma^{2}$ as a function of the effective damping $\gamma$ evaluated in the perturbative regime with strong asymmetry $\kappa=0.01$. Above a critical value $\gamma_{c}=0.285 \omega_{0}$ no positive values for $J_{1}$ are found. (b) Scaling behavior of the minimum achievable temperature $T_{\min }$ with the cooling mechanism as a function of $\gamma$. The data points agree well with the scaling behavior $\propto \sqrt{\gamma}$.

the $T \rightarrow 0$ limit can only be achieved for $\gamma \rightarrow 0$ in infinite time, in agreement with Nernst's principle. In addition (data not shown here), the corresponding driving frequency approaches (from below) $\Omega \rightarrow \omega_{0}$. From the plot, it is also evident that the scaling behavior with $\gamma$ exhibits a $\sqrt{\gamma}$ dependence. Note that a similar scaling was found by Freitas et al. [42] in a different context: static system-bath ohmic coupling and in the presence of an external parametric drive of the oscillator frequency. We remark that the underlying mechanism discussed here is different, since it relies on temporal variation of the system-bath couplings and ratchet effect without any external field.

To further exploit the ratchet-induced cooling mechanism discussed until now for isothermal reservoirs, we consider the two reservoirs at different temperatures. In particular, we choose symmetric deviations from the isothermal situation with $T_{1,2}=T \mp \Delta T / 2$, where $T$ represents the average temperature and $\Delta T$ the temperature gradient. Here, in the presence of finite thermal gradient $\Delta T \neq$ 0 , one can look for refrigeration properties [9,80-84], i.e., heat extracted from the colder reservoir thanks to the ratchet dynamics induced by the coupling drives. To quantify this effect, we consider the figure of merit

$$
\eta(\Omega, \Delta T / T)=\frac{J_{1}(\Omega, \Delta T / T)}{\left|J_{1}(\Omega, \Delta T / T)+J_{2}(\Omega, \Delta T / T)\right|},
$$

which represents the so-called coefficient of performance (COP) of refrigerators at a fixed average temperature $T$. This quantity is plotted in Fig. 5(a) as a function of external frequency $\Omega$ and relative temperature gradient $\Delta T / T$. As an average temperature we have chosen $T=0.107 \omega_{0}$, which is the value that maximizes $\eta(\Omega, \Delta T=0)$, i.e., the ratio $J_{1} / P$ in the isothermal case. As one can see, the colder $v=1$ reservoir can be cooled $\left(J_{1}>0\right)$ in a relatively large parameter region of the $(\Omega-\Delta T / T)$ plane (see the colored area).

In order to quantify the efficiency, in Fig. 5(b) we plot the function $\eta$ normalized to the Carnot value for
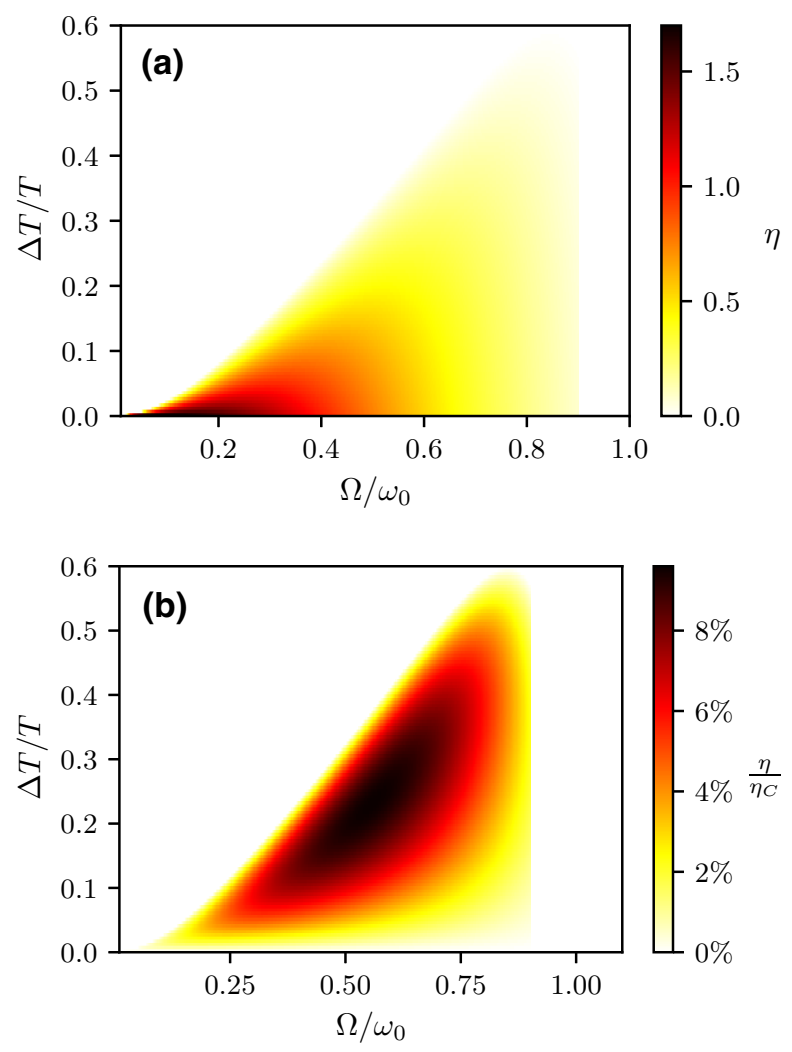

FIG. 5. Ratchet-induced refrigeration. (a) Density plot of $\eta=$ $J_{1} /\left|J_{1}+J_{2}\right|$ as a function of $\Omega / \omega_{0}$ and relative temperature variation $\Delta T / T$ with average temperature $T=0.107 \omega_{0}$. (b) Density plot of the ratio $\eta / \eta_{C}$ (in percentages). Other parameters are $\gamma=0.01 \omega_{0}$ and $\kappa=0.01$. 
refrigerators [9]:

$$
\eta_{C}=\frac{T_{1}}{T_{2}-T_{1}}=-\frac{1}{2}+\frac{T}{\Delta T} .
$$

Here, it is possible to achieve value of the COP up to about $10 \%$ of $\eta_{C}$. Remarkably, such values are obtained in the nonlinear regime and at finite frequency (with $\Omega \sim 0.6 \omega_{0}$ ). In passing, we mention that even higher values of this ratio can be achieved, decreasing the effective damping $\gamma$, although the magnitude of the heat currents will be smaller.

\section{B. Beyond dynamical constraints}

The results discussed so far have been obtained within the particular choice (40) for the drives, which allows for an exact solution of the problem. At first sight this could seem a very peculiar case. However, in the following we demonstrate that the possibility of performing a fundamental quantum thermodynamic task is more general and goes beyond the above assumption. In order to corroborate this point and to verify the stability of the dynamically induced cooling phenomenon, we consider different timedependent drives outside the constraint class. Among all possibilities, as an illustrative example, we choose

$$
g_{1}(t)=\cos (\Omega t), \quad g_{2}(t)=1 .
$$

Here one of the two couplings oscillates [Fourier components $g_{n, 1}=\left(\delta_{n, 1}+\delta_{n,-1}\right) / 2$ ], while the other is constant $\left(g_{n, 2}=\delta_{n, 0}\right)$. Despite its simplicity, we also expect here the induced ratchetlike phenomenon, which is one of the key ingredients to obtain cooling by modulating the systembath couplings. The spectral densities of the two baths are again ohmic with damping $\gamma_{1}$ and $\gamma_{2}$. Also, in this case we define an effective damping strength $\gamma=\operatorname{Max}\left[\gamma_{1}, \gamma_{2}\right]$ and the dimensionless parameter that governs the asymmetry $\kappa=\gamma_{1} / \gamma_{2}$.

The choice (51) is also motivated by the fact that, for $\kappa \rightarrow 0$, the unconstrained model falls in the same universality class as that of Eqs. (44). Indeed, as shown in Appendix E, up to linear order in $\kappa$ the heat currents are equal to the perturbative expressions given in Eqs. (47)-(48). More generally, we have evaluated, at any order in $\kappa$, the heat currents, by first solving, via exact diagonalization, the algebraic equations (31) for the Floquet coefficients $G_{m}(\omega)$. Indeed, in this general case, several Floquet components, other than the static one, will give finite contributions. The heat currents are then obtained by inserting the results of $G_{m}(\omega)$ and the couplings (51) into the general expression in Eq. (35).

The numerical results are reported in Fig. 6 for different values of $\kappa$ (red triangles in the plots). In Fig. 6(a) we show $\operatorname{Max}\left[J_{1}\right] / P$, that is, the maximum positive value of $J_{1}$ normalized to the corresponding supplied power, in the isothermal case, in analogy with Fig. 3(b). Our results
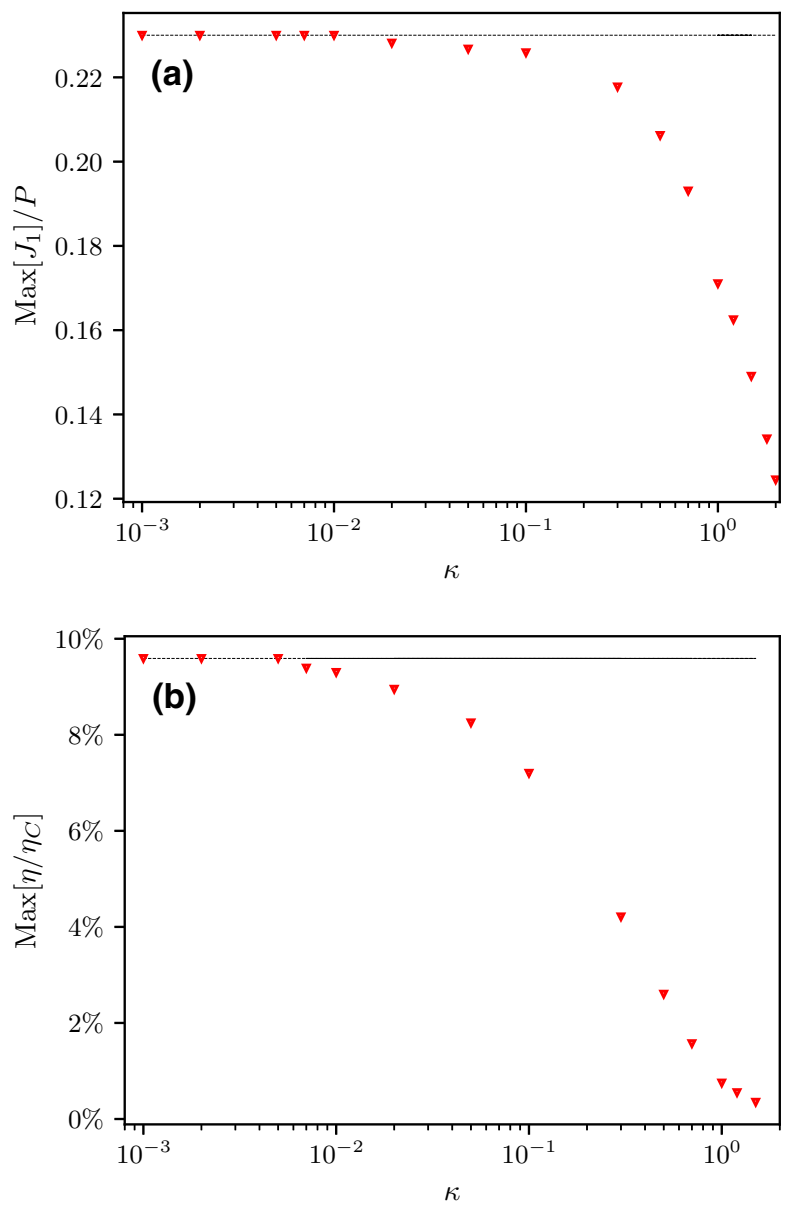

FIG. 6. Ratchet-induced cooling beyond the dynamical constraint. (a) Maximum positive heat current normalized to the corresponding supplied power $\operatorname{Max}\left[J_{1}\right] / P$ as a function of $\kappa=$ $\gamma_{1} / \gamma_{2}$ in the isothermal case $T_{1}=T_{2}$. (b) Maximum value of the COP normalized to the Carnot bound $\operatorname{Max}\left[\eta / \eta_{C}\right]$ in percentages, as a function of $\kappa$, considering finite temperature gradient $\Delta T \neq 0$. The black dashed lines correspond to the asymptotic value obtained within the perturbative expansion. Note that the data points tend to saturate to the perturbative results for small $\kappa$ values. Other parameters are $\gamma=\operatorname{Max}\left[\gamma_{1}, \gamma_{2}\right]=0.01 \omega_{0}$ and $\omega_{c}=500 \omega_{0}$.

demonstrate that it is possible, also in this case, to obtain a positive value of $J_{1}$ and, thus, cooling induced by suitably engineered temporal modulation of the driven couplings. Here, the effect extends in a wider region of the asymmetry parameter $\kappa$ and still a finite (although small) effect is visible for $\kappa \geq 1$ also. The dashed line in the plot represents the asymptotic value obtained in the perturbative regime at $\kappa \ll 1$ that corresponds to the value also reported in Fig. 3(b). In Fig. 6(b) we consider finite thermal gradients $\Delta T \neq 0$, looking for refrigeration properties. Here, we report the maximum value of $\eta / \eta_{C}$ in percentages. Again, from this figure one can deduce that refrigeration associated with the $v=1$ reservoir is also a robust 
feature beyond the constraint (40), and the optimal working regime (within this universality class) is obtained for small values of $\kappa$, i.e., in the case of strong asymmetry between the two bath couplings (but only in one direction, namely for $\gamma_{1} \ll \gamma_{2}$ ). This example proves the robustness of the discussed phenomenon beyond the particular choice of time-dependent drives and interestingly opens the possibility to study more complicated situations where the refrigeration response could be improved.

\section{Dynamical heat rectification}

Before closing, we comment on another relevant aspect for quantum devices, i.e., the possibility to achieve rectification of heat current flows [45,46,85-94]. It has recently been shown that heat rectification can be obtained in a linear system (as a QHO) by relying on external timedependent forces $[78,95]$. Here we demonstrate that a dynamically induced heat rectification is also present without additional external fields, but only in the presence of time-dependent system-bath couplings. To this end, we focus on the heat current $J_{1}$, associated with the $v=1$ reservoir, in the presence of a thermal gradient $\Delta T$ on top of an average temperature $T$. In order to quantify heat rectification, we define two configurations with interchanged temperatures, namely,

$$
\begin{aligned}
& J_{1}^{f}(T, \Delta T)=J_{1}\left(T_{1}=T+\Delta T / 2, T_{2}=T-\Delta T / 2\right), \\
& J_{1}^{b}(T, \Delta T)=J_{1}\left(T_{1}=T-\Delta T / 2, T_{2}=T+\Delta T / 2\right) .
\end{aligned}
$$

A useful figure of merit for rectification is then represented by the ratio

$$
R \equiv-\frac{J_{1}^{f}(T, \Delta T)}{J_{1}^{b}(T, \Delta T)}
$$

Here, we adopted the standard notation $f$ for forward and $b$ for backward, typically used in the presence of only a static thermal gradient $[45,46,85]$, where forward and backward represent the directions of the heat fluxes and $R \geq 0$. The value $R=1$ indicates no heat rectification. Note that in our dynamically driven case, forward or backward does not necessarily imply a fixed direction of flow. Indeed, it is possible to have both heat currents $J_{1}^{f}$ and $J_{1}^{b}$ flowing in the same direction with then $R<0$. We have already met this situation in the isothermal case $\Delta T=0$, where a finite heat current is in general present in spite of the lack of thermal gradient, due to the asymmetric modulation of the couplings. This is a kind of Peltier effect, but of dynamical origin. In this case forward and backward heat currents coincide with $R=-1$. In general, by varying the temperature gradient and the driving frequency $\Omega$, the amplitudes and direction of the two heat currents change,
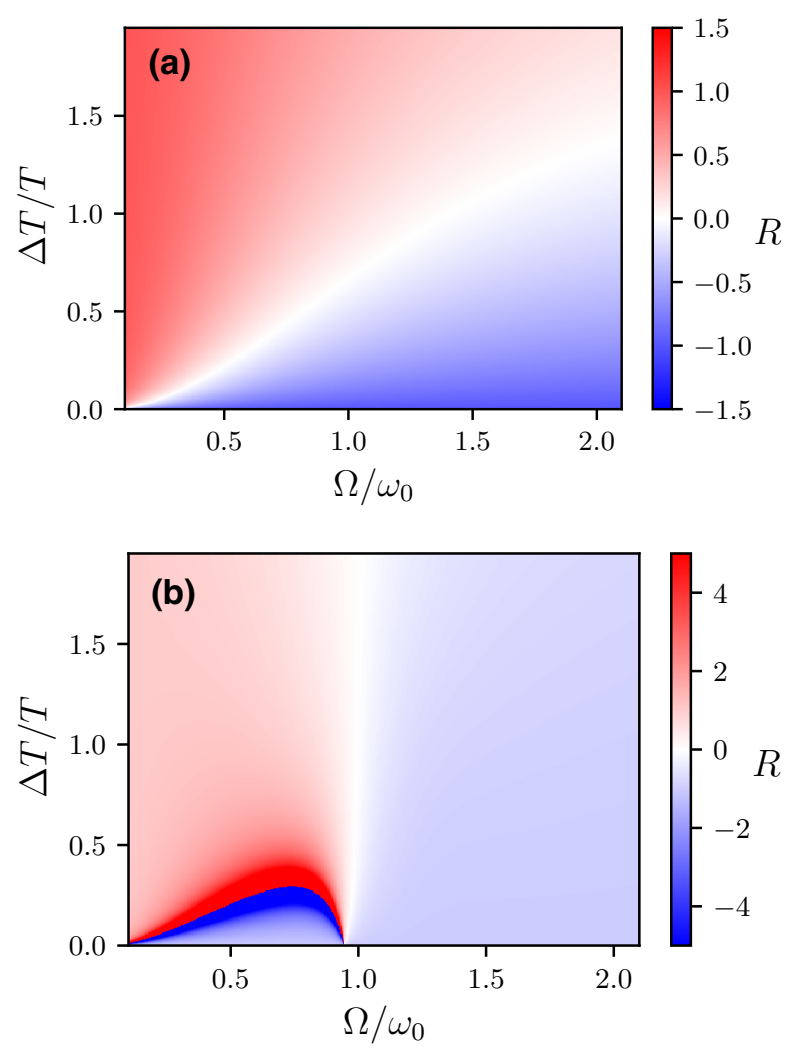

FIG. 7. Dynamical heat rectification. Density plots of the ratio $R$ in Eq. (53) between forward and backward heat currents as a function of external frequency $\Omega / \omega_{0}$ and normalized temperature gradient $\Delta T / T$. (a) Classic regime, with average temperature $T=10 \omega_{0}$. (b) Deep quantum regime with $T=0.15 \omega_{0}$. Other parameters are $\kappa=0.01$ and $\gamma=0.01 \omega_{0}$.

resulting in $|R| \neq 1$. The situation where the forward or backward configuration is completely blocked is indicated by $|R| \rightarrow 0$ or $|R| \rightarrow \infty$, respectively.

The ratio $R$ is reported in Fig. 7 as a function of the driving frequency $\Omega / \omega_{0}$ and normalized temperature gradient $\Delta T / T$. As an example, we evaluated $R$ in the perturbative regime with $\kappa \ll 1$, using Eq. (47). Figure 7(a) shows the classical regime $T \gg \omega_{0}$. Here, for $\Delta T \rightarrow 0$, the dynamical Peltier contribution is the dominant one: both $J_{1}^{f}$ and $J_{1}^{b}$ start negative with $R \rightarrow-1$. Increasing $\Delta T, J_{1}^{b}$ assumes larger negative values, while $J_{1}^{f}$ decreases, until it changes sign. Therefore, it is possible to block the heat current in the forward configuration $(R=0$; white regions in the density plot). Note that the reverse situation of blocking the backward heat current $(|R| \rightarrow \infty)$ is here never possible: indeed, in Fig. 7(a) it is always $|R| \leq 1$.

A much more versatile situation, instead, can be obtained in the quantum non-Markovian regime at low average temperature $T \ll \omega_{0}$, as shown in Fig. 7(b). Here, we have two nonoverlapping regions that correspond to two orthogonal cases. The first one, with $\Omega \approx \omega_{0}$ (white area in the plot), has $R \rightarrow 0$, signaling full blocking of $J_{1}^{f}$, 
similar to the classical case. On the other hand, in the second region (left bottom part of the density plot) one can achieve the full block of the backward heat current $J_{1}^{b}$ $(|R| \rightarrow \infty)$. Indeed, increasing $\Delta T / T$, the backward current $J_{1}^{b}$ changes sign, passing from positive to negative values along the line situated between the two strong blue and red color regions. Meanwhile, $J_{1}^{f}$ always remains positive. Importantly, these features are present in the same parameter regions with $\Omega<\omega_{0}$, where finite refrigeration is obtained in response to the driven couplings [see Fig. 5(a)].

In summary, our system is much more versatile in the quantum regime, since in such a case it is possible, by modulating the system-bath couplings, to create a heat rectifier that can switch by blocking either the forward or backward current by simply varying the driving frequency $\Omega$.

\section{CONCLUSIONS}

The extension of thermodynamics to small, quantum systems, challenges the usual paradigms of traditional thermodynamics, like local thermal equilibrium, weak systemreservoir coupling, and Markovianity. As usual, when facing the quantum world, even the most intuitive concepts should be carefully reexamined. For instance, one might reasonably argue that a purely dissipative effect is associated with switching on and off the couplings to reservoirs in a nonadiabatic way, as required in any finite-time thermodynamic cycle. In contrast to this common belief, here we have shown that basic thermodynamic tasks can be performed by periodically modulating in a suitable way only the couplings to the baths. Indeed, asymmetric baths can be suitably engineered to induce cooling, refrigeration, and ideal heat rectification along a direction that can be reversed simply by tuning the modulation frequency.

We have described the quantum baths by the CaldeiraLeggett model, so that the system's dynamics and thermodynamics can be investigated without resorting to the overdamped limit, to suitable master equations, or other approximations. The usefulness of this general framework has been tested here for the case where the system is a single harmonic oscillator, the bath is ohmic, and only the system-bath couplings are time dependent. To summarize, we have schematically reported our main results and their working parameter regimes in Table I. On the other hand, our study paves the way to several possible generalizations. For instance, since the most intriguing results have been obtained in the non-Markovian, quantum regime, one could consider nonohmic baths where non-Markovian effects are also present in the classical, high-temperature regime, in order to disentangle the relevance of non-Markovian and quantum effects. The main results have been obtained in the simplest configuration, where the QHO is coupled to two reservoirs. The extension to multiterminal configurations could be exploited
TABLE I. Summary of main results and parameter regimes obtained in the strongly asymmetric case $\kappa \ll 1$ with $\gamma \ll 1$. Recall that $R=1$ means no heat rectification.

\begin{tabular}{lccc}
\hline \hline & $\Omega$ & $T$ & $\Delta T$ \\
\hline Cooling $\left(J_{1}>0\right)$ & $<\omega_{0}$ & $\ll \omega_{0}$ & 0 \\
Refrigerator & $<\omega_{0}$ & $\ll \omega_{0}$ & $>0$ \\
Heat rectification & Any & Any & $>0$ \\
Blocking forward current $R=0$ & $<\omega_{0}$ & $\ll \omega_{0}$ & $>0$ \\
& Any & $>\omega_{0}$ & $f(\Omega)$ \\
Blocking backward current $R=\infty$ & $<\omega_{0}$ & $\ll \omega_{0}$ & $>0$ \\
\hline \hline
\end{tabular}

to obtain independent control of work and heat flows in different input and output channels, thus limiting heating of working resources and achieving optimally engineered thermal routing [15,46,96-100], thermal transistors [14], cooling by heating powered by a third, photonic reservoir [101-103], and boosted energy conversion [104-106]. Moreover, one could consider the joint effect of modulating the bath couplings, and in addition driving the system, to investigate whether these external controls could cooperate in order to enhance the performance of refrigeration. The same problems could be addressed for heat engines, and in both cases the developed formalism is ideally suited to apply optimal control techniques [62,107-109]. In particular, it would be interesting to reconsider the results recently obtained [30] for isothermal heat engines, where in the antiadiabatic limit the ideal efficiency is approached with finite output power and vanishingly small relative power fluctuations. The intriguing question here is whether the simultaneous achievement of the three desiderata of a heat engine (efficiency close to the Carnot efficiency, high output power, and constancy of the power output) is also possible for standard heat engines operating with two or more heat baths at different temperatures. Further generalizations of our approach could be obtained by considering a more complex working medium [17,110-114], like coupled oscillators and, with a considerably higher numerical effort, nonlinear oscillators and qubit systems.

\section{ACKNOWLEDGMENTS}

This work is supported by the "Dipartimento di Eccellenza MIUR 2018-2022". M.C. acknowledges the QuantEraNet project "SuperTop". G.B. acknowledges financial support from the INFN through the project "QUANTUM".

\section{APPENDIX A: OUT-OF-EQUILIBRIUM GREEN'S FUNCTION AND FLOQUET COEFFICIENTS}

In this part we derive the algebraic set of equations for the Floquet coefficients $\tilde{G}_{m}(\omega)$ quoted in Eq. (31). We start from the differential equation in Eq. (26) written in the 
following compact form

$$
\begin{aligned}
& \frac{\partial^{2}}{\partial t^{2}} G\left(t, t^{\prime}\right)+\omega_{0}^{2} G\left(t, t^{\prime}\right) \\
& \quad+\int_{-\infty}^{+\infty} d s\left[u(t, s) G\left(s, t^{\prime}\right)+v(t, s) \frac{\partial}{\partial s} G\left(s, t^{\prime}\right)\right] \\
& \quad=\delta\left(t-t^{\prime}\right) .
\end{aligned}
$$

Here, we have introduced the bath kernels

$$
\begin{aligned}
& u(t, s)=\sum_{\nu=1}^{N} g_{\nu}(t) \gamma_{\nu}(t-s) \frac{d}{d s} g_{v}(s), \\
& v(t, s)=\sum_{\nu=1}^{N} g_{\nu}(t) \gamma_{\nu}(t-s) g_{\nu}(s)
\end{aligned}
$$

expressed in terms of the periodic couplings

$$
g_{\nu}(t)=\sum_{n=-\infty}^{+\infty} g_{n, \nu} e^{-i n \Omega t}, \quad \Omega=\frac{2 \pi}{\mathcal{T}} .
$$

We recall that at long times it holds the property

$$
G\left(t, t^{\prime}\right)=\int_{-\infty}^{+\infty} \frac{d \omega}{2 \pi} e^{-i \omega\left(t-t^{\prime}\right)} \tilde{G}(t, \omega),
$$

where $\tilde{G}(t, \omega)$ is a periodic function of $t$ with period $\mathcal{T}$ :

$$
\tilde{G}(t, \omega)=\sum_{m=-\infty}^{+\infty} \tilde{G}_{m}(\omega) e^{-i m \Omega t}
$$

A similar representation can also be carried out for the kernels $u(t, s)$ and $v(t, s)$ :

$$
\begin{aligned}
& u(t, s)=\int_{-\infty}^{+\infty} \frac{d \omega}{2 \pi} e^{-i \omega(t-s)} \tilde{u}(t, \omega), \\
& v(t, s)=\int_{-\infty}^{+\infty} \frac{d \omega}{2 \pi} e^{-i \omega(t-s)} \tilde{v}(t, \omega),
\end{aligned}
$$

with

$$
\tilde{u}(t, \omega)=\sum_{m=-\infty}^{+\infty} \tilde{u}_{m}(\omega) e^{-i m \Omega t}
$$

and

$$
\tilde{v}(t, \omega)=\sum_{m=-\infty}^{+\infty} \tilde{v}_{m}(\omega) e^{-i m \Omega t}
$$

Now, we transform differential equation (A1) into a set of coupled algebraic equations. This can be done by inserting representations (A5)-(A6) and those for the kernels in Eqs. (A7)-(A10) into Eq. (A1). As a representative example, we quote the expression for the term associated with the damping contribution

$$
\mathcal{D}\left(t, t^{\prime}\right)=\int_{-\infty}^{+\infty} d s\left[u(t, s) G\left(s, t^{\prime}\right)+v(t, s) \frac{\partial}{\partial s} G\left(s, t^{\prime}\right)\right] .
$$

We have

$$
\begin{aligned}
\mathcal{D}\left(t, t^{\prime}\right)= & \int_{-\infty}^{+\infty} \frac{d \omega}{2 \pi} e^{-i \omega\left(t-t^{\prime}\right)} \sum_{m=-\infty}^{+\infty} \tilde{G}_{m}(\omega) \tilde{k}(t, \omega+m \Omega) \\
& \times e^{-i m \Omega t}
\end{aligned}
$$

with

$$
\tilde{k}(t, \omega)=\tilde{u}(t, \omega)-i \omega \tilde{v}(t, \omega) .
$$

Expressing $\tilde{k}(t, \omega)$ with the series (A9)-(A10) we obtain

$$
\begin{gathered}
\mathcal{D}\left(t, t^{\prime}\right)=\int_{-\infty}^{+\infty} \frac{d \omega}{2 \pi} e^{-i \omega\left(t-t^{\prime}\right)} \sum_{m_{1}=-\infty}^{+\infty} \sum_{m_{2}=-\infty}^{+\infty} \\
\times \tilde{G}_{m_{1}}(\omega) \tilde{k}_{m_{2}}\left(\omega+m_{1} \Omega\right) e^{-i\left(m_{1}+m_{2}\right) \Omega t}
\end{gathered}
$$

with

$$
\tilde{k}_{m}(\omega)=\tilde{u}_{m}(\omega)-i \omega \tilde{v}_{m}(\omega)
$$

Explicitly, we have

$\tilde{k}_{m}(\omega)=-i \sum_{\nu=1}^{N} \sum_{n=-\infty}^{+\infty} g_{n, \nu} g_{m-n, \nu} \times(n \Omega+\omega) \tilde{\gamma}_{\nu}(\omega+n \Omega)$,

where

$$
\tilde{\gamma}_{v}(\omega)=\int_{-\infty}^{+\infty} d t e^{i \omega t} \gamma_{v}(t)
$$

Following similar steps for all the terms in Eq. (A1) we obtain the set of algebraic equations

$$
\begin{aligned}
& {\left[\omega_{0}^{2}-(\omega+m \Omega)^{2}\right] \tilde{G}_{m}(\omega)} \\
& +\sum_{n=-\infty}^{+\infty} \tilde{k}_{n}[\omega+(m-n) \Omega] \tilde{G}_{m-n}(\omega) \\
& \quad=\delta_{m, 0} .
\end{aligned}
$$

By now introducing the "static" retarded Green function in the spectral domain

$$
\chi_{0}(\omega)=-\frac{1}{\omega^{2}-\omega_{0}^{2}-\tilde{k}_{0}(\omega)},
$$


which contains the $n=0$ component of the bath kernel $\tilde{k}_{n}(\omega)$, we rewrite Eq. (A17) in the compact form

$$
\begin{gathered}
\tilde{G}_{m}(\omega)+\chi_{0}(\omega+m \Omega) \sum_{n=-\infty, n \neq 0}^{+\infty} \tilde{k}_{n}(\omega+(m-n) \Omega) \tilde{G}_{m-n}(\omega) \\
=\chi_{0}(\omega) \delta_{m, 0},
\end{gathered}
$$

as reported in the main text.

\section{APPENDIX B: EXPLICIT EXPRESSIONS FOR AVERAGE HEAT CURRENTS}

In this appendix we derive the explicit expressions for the average heat current $J_{v}$ quoted in Eq. (35). We start by considering Eq. (34) for the time-dependent heat current $J_{v}(t)$ and we separate it into two contributions:

$$
J_{v}(t)=J_{v}^{(a)}(t)+J_{v}^{(b)}(t)
$$

with

$$
\begin{aligned}
J_{v}^{(a)}(t)= & -\frac{g_{v}(t)}{m} \int_{-\infty}^{+\infty} d t^{\prime} G\left(t, t^{\prime}\right) g_{v}\left(t^{\prime}\right) \dot{\mathcal{L}}_{v}^{(+)}\left(t-t^{\prime}\right), \\
J_{v}^{(b)}(t)= & -\frac{2 g_{v}(t)}{m^{2}} \int_{-\infty}^{t} d s \int_{-\infty}^{+\infty} d t_{1} \\
& \times \int_{-\infty}^{+\infty} d t_{2} g_{v}(s) \dot{\mathcal{L}}_{v}^{(-)}(t-s) G\left(t, t_{1}\right) G\left(s, t_{2}\right) \\
& \times \sum_{v_{1}=1}^{N} g_{v_{1}}\left(t_{1}\right) g_{v_{1}}\left(t_{2}\right) \mathcal{L}_{v_{1}}^{(+)}\left(t_{1}-t_{2}\right) .
\end{aligned}
$$

We recall that the average heat currents $J_{v}$ are obtained from $J_{v}^{(a / b)}(t)$ after performing the cycle average over the period $\mathcal{T}$ :

$$
J_{v}^{(a / b)}=\frac{1}{\mathcal{T}} \int_{0}^{\mathcal{T}} d t J_{v}^{(a / b)}(t)
$$

with $J_{v}=J_{v}^{(a)}+J_{v}^{(b)}$.

To proceed further, we rewrite $J_{v}^{(a / b)}(t)$ upon a change of variables as

$$
\begin{aligned}
& J_{v}^{(a)}(t)=-\int_{-\infty}^{+\infty} d \tau \dot{\mathcal{L}}_{v}^{(+)}(\tau) M_{v}^{(a)}(t, t-\tau), \\
& J_{v}^{(b)}(t)=-\int_{0}^{+\infty} d \tau \dot{\mathcal{L}}_{v}^{(-)}(\tau) M_{v}^{(b)}(t, t-\tau),
\end{aligned}
$$

with

$$
M_{v}^{(a)}(t, t-\tau)=\frac{1}{m} g_{v}(t) g_{v}(t-\tau) G(t, t-\tau),
$$

$$
\begin{aligned}
M_{v}^{(b)}(t, t-\tau)= & \frac{2}{m^{2}} g_{v}(t) g_{v}(t-\tau) \\
& \times \int_{-\infty}^{+\infty} d t_{1} \int_{-\infty}^{+\infty} d t_{2} G\left(t, t_{1}\right) G\left(t-\tau, t_{2}\right) \\
& \times \sum_{v_{1}=1}^{N} g_{v_{1}}\left(t_{1}\right) g_{v_{1}}\left(t_{2}\right) \mathcal{L}_{v_{1}}^{(+)}\left(t_{1}-t_{2}\right) .
\end{aligned}
$$

Note that the $t$ dependence is now only present in the functions $M^{(a / b)}(t, t-\tau)$. Therefore, the average (B3) is

$$
\begin{aligned}
& J_{v}^{(a)}=-\int_{-\infty}^{+\infty} d \tau \dot{\mathcal{L}}_{v}^{(+)}(\tau) M_{v}^{(a)}(\tau), \\
& J_{v}^{(b)}=-\int_{0}^{+\infty} d \tau \dot{\mathcal{L}}_{v}^{(-)}(\tau) M_{v}^{(b)}(\tau),
\end{aligned}
$$

with

$$
M_{v}^{(a / b)}(\tau)=\frac{1}{\mathcal{T}} \int_{0}^{\mathcal{T}} d t M_{v}^{(a / b)}(t, t-\tau) .
$$

We now evaluate $M_{v}^{(a / b)}(\tau)$. First we insert representations (4) and (30) into Eqs. (B5), obtaining

$$
\begin{aligned}
M_{v}^{(a)}(t, t-\tau)= & \sum_{m_{1}=-\infty}^{+\infty} e^{-i m_{1} \Omega t} \sum_{n_{1}, n_{2}=-\infty}^{+\infty} g_{n_{1}, \nu} g_{n_{2}, v} \\
& \times \int_{-\infty}^{+\infty} \frac{d \omega}{2 \pi m} \tilde{G}_{m_{1}-\left(n_{1}+n_{2}\right)}(\omega) e^{i\left(n_{2} \Omega-\omega\right) \tau},
\end{aligned}
$$

$$
\begin{aligned}
& M_{v}^{(b)}(t, t-\tau) \\
& =\sum_{m_{1}, m_{2}=-\infty}^{+\infty} e^{-i m_{1} \Omega t} \sum_{n_{1} \cdots n_{4}=-\infty}^{+\infty} g_{n_{1}, \nu} g_{n_{2}, v} \sum_{\nu_{1}=1}^{N} g_{n_{3}, \nu_{1}} g_{n_{4}, \nu_{1}} \\
& \quad \times \int_{-\infty}^{+\infty} \frac{d \omega}{\pi m^{2}} \tilde{G}_{m_{1}-\left(m_{2}+n_{\mathrm{tot} t}\right)}(\omega) \tilde{G}_{m_{2}}\left[\left(n_{3}+n_{4}\right) \Omega-\omega\right] \\
& \quad \times \widetilde{\mathcal{L}}_{v_{1}}^{(+)}\left(\omega-n_{3} \Omega\right) e^{i\left[\left(n_{2}+n_{3}+n_{4}\right)+m_{2}\right] \Omega \tau} e^{-i \omega \tau},
\end{aligned}
$$

where $n_{\text {tot }}=n_{1}+n_{2}+n_{3}+n_{4}$. In the above expressions we have introduced the Fourier transform of the symmetric and antisymmetric parts of the bath correlators $\mathcal{L}_{v}^{( \pm)}(t)$ in Eq. (15). They are defined as

$$
\widetilde{\mathcal{L}}_{v}^{( \pm)}(\omega)=\int_{-\infty}^{\infty} d t \mathcal{L}_{v}^{( \pm)}(t) e^{i \omega t},
$$

and they have an explicit form in terms of the bath spectral densities $\mathcal{J}_{\nu}(\omega)$ :

$$
\widetilde{\mathcal{L}}_{v}^{(+)}(\omega)=\mathcal{J}_{v}(\omega) \operatorname{coth}\left(\frac{\omega}{2 T_{v}}\right)
$$




$$
\widetilde{\mathcal{L}}_{v}^{(-)}(\omega)=i \mathcal{J}_{v}(\omega)
$$

with $\mathcal{J}_{v}(\omega)=-\mathcal{J}_{v}(-\omega)$. We now perform the cycle average (B7), which yields

$$
\begin{aligned}
M_{\nu}^{(a)}(\tau)= & \sum_{n_{1}, n_{2}=-\infty}^{+\infty} g_{n_{1}, v} g_{n_{2}, v} \int_{-\infty}^{+\infty} \frac{d \omega}{2 \pi m} e^{i\left(n_{2} \Omega-\omega\right) \tau} \tilde{G}_{-\left(n_{1}+n_{2}\right)}(\omega), \\
M_{\nu}^{(b)}(\tau)= & \sum_{m_{1}=-\infty}^{+\infty} \sum_{n_{1} \cdots n_{4}=-\infty}^{+\infty} g_{n_{1}, \nu} g_{n_{2}, v} \sum_{\nu_{1}=1}^{N} g_{n_{3}, \nu_{1}} g_{n_{4}, \nu_{1}} \int_{-\infty}^{+\infty} \frac{d \omega}{\pi m^{2}} \tilde{G}_{-\left(m_{1}+n_{\mathrm{tot}}\right)}(\omega) \tilde{G}_{m_{1}}\left(\left(n_{3}+n_{4}\right) \Omega-\omega\right) \\
& \times \tilde{\mathcal{L}}_{\nu_{1}}^{(+)}\left(\omega-n_{3} \Omega\right) e^{i\left[\left(n_{2}+n_{3}+n_{4}\right)+m_{1}\right] \Omega \tau} e^{-i \omega \tau} .
\end{aligned}
$$

Inserting these expressions into Eqs. (B6) we perform the $\tau$ integrals by using Eqs. (B11) and (B12). Note that $M_{v}^{(b)}(\tau)=$ $M_{v}^{(b)}(-\tau)$. The final result for the average heat currents, uponsumming the two contributions, is reported in Eq. (35) in the main text.

Starting from Eq. (25) and following similar steps we also obtain the average power associated with the $v$ th bath:

$$
\begin{aligned}
P_{v}= & \Omega \sum_{n_{1}, n_{2}=-\infty}^{+\infty} n_{1} g_{n_{1}, v} g_{n_{2}, v} \int_{-\infty}^{+\infty} \frac{d \omega}{2 \pi m}\left\{i \mathcal{J}_{\nu}(\omega) \operatorname{coth}\left(\frac{\omega}{2 T_{v}}\right) \tilde{G}_{-\left(n_{1}+n_{2}\right)}\left(\omega+n_{2} \Omega\right)+\sum_{v_{1}=1}^{N} \sum_{m_{1}=-\infty}^{+\infty} \sum_{n_{3}, n_{4}=-\infty}^{+\infty} g_{n_{3}, \nu_{1}} g_{n_{4}, \nu_{1}} \frac{\mathcal{J}_{\nu_{1}}(\omega)}{m}\right. \\
& \left.\times \operatorname{coth}\left(\frac{\omega}{2 T_{v_{1}}}\right) \mathcal{J}_{\nu}\left[\omega-\Omega\left(n_{2}+n_{4}+m_{1}\right)\right] \tilde{G}_{m_{1}}\left(-\omega+n_{4} \Omega\right) \tilde{G}_{-\left(n_{\mathrm{tot}}+m_{1}\right)}\left(\omega+n_{3} \Omega\right)\right\}
\end{aligned}
$$

\section{APPENDIX C: AVERAGE HEAT CURRENTS IN THE CLASSICAL REGIME}

Here, we demonstrate that in the classical regime $\left(T \gg \omega_{0}\right)$, the average heat currents for isothermal baths $\left(T_{v}=T\right)$ are always dissipative $\left(J_{v}<0\right)$. To this end, we focus on the case where the time-dependent couplings are linked by constraint (40). We then start from the heat current expressions given in Eq. (43). In the classical limit we substitute $\operatorname{coth}(\omega / 2 T) \rightarrow 2 T / \omega$, obtaining

$$
\begin{aligned}
J_{v}= & T \gamma_{\nu} \int_{-\infty}^{+\infty} \frac{d \omega}{\pi}\left\{\sum_{n=-\infty}^{+\infty}\left|g_{n, v}\right|^{2} \omega \operatorname{Im} \chi_{0}(\omega+n \Omega)-\sum_{n_{1}, n_{3}, n_{4}=-\infty}^{+\infty} g_{n_{1}, \nu} g_{-\left(n_{1}+n_{3}+n_{4}\right), v} \sum_{\nu_{1}=1}^{N} \gamma_{\nu_{1}} g_{n_{3}, \nu_{1}} g_{n_{4}, \nu_{1}}\right. \\
& \left.\times\left[\omega+\Omega\left(n_{1}+n_{3}\right)\right]^{2} \chi_{0}\left(-\omega+n_{4} \Omega\right) \chi_{0}\left(\omega+n_{3} \Omega\right)\right\} .
\end{aligned}
$$

Note that these expressions are well behaved at large $\omega$ and therefore we have safely taken the $\omega_{c} \rightarrow \infty$ limit. We recall that $\operatorname{Im} \chi_{0}(\omega)$ has the property

$$
\operatorname{Im} \chi_{0}(\omega)=\gamma \omega\left|\chi_{0}(\omega)\right|^{2}
$$

with

$$
\chi_{0}(\omega)=\frac{-1}{\omega^{2}-\omega_{0}^{2}+i \omega \gamma}
$$

Expression (C1) can be rewritten by performing, in the second term, the change of variables $\left[\omega+\Omega\left(n_{1}+n_{3}\right)\right] \rightarrow \omega$ and $n_{1}+n_{2}+n_{3} \rightarrow n_{3}$ and by exploiting the relation

$$
\sum_{\nu=1}^{N} \gamma_{\nu} \sum_{n=-\infty}^{+\infty} g_{n, \nu} g_{-n+m, \nu}=\gamma \delta_{m, 0},
$$


which derives from link (40). We have the final result

$$
J_{\nu}=-T \gamma_{\nu} \gamma \Omega^{2} \sum_{n=-\infty}^{+\infty} n^{2}\left|g_{n, v}\right|^{2} \int_{-\infty}^{+\infty} \frac{d \omega}{\pi} \omega^{2}\left|\chi_{0}(\omega)\right|^{2}
$$

which is always negative. We can then conclude that in the classical regime for isothermal ohmic baths, it is impossible to obtain any cooling effect.

\section{APPENDIX D: EXPLICIT EXPRESSIONS IN THE PERTURBATIVE REGIME}

In this appendix, we derive the perturbative expressions for the average heat currents given in Eqs. (47)-(48).

We start by commenting on the iterative procedure used in perturbative schemes in order to solve the algebraic equations (31) for the Floquet coefficients. The procedure starts, as a zeroth step, by choosing $\tilde{G}_{m}(\omega)=\delta_{m, 0} \chi_{0}(\omega)$, then by inserting it into Eq. (31). One finds the first corrections

$$
\tilde{G}_{m \neq 0}(\omega)=-\chi_{0}(\omega+m \Omega) \tilde{k}_{m}(\omega) \chi_{0}(\omega) .
$$

The second iteration is obtained by dressing $\tilde{G}_{0}(\omega)$ with

$$
\begin{aligned}
\tilde{G}_{0}(\omega)= & \chi_{0}(\omega)+\chi_{0}(\omega) \sum_{n \neq 0} \tilde{k}_{n}(\omega-n \Omega) \\
& \times \chi_{0}(\omega-n \Omega) \tilde{k}_{-n}(\omega) \chi_{0}(\omega),
\end{aligned}
$$

and so on and so forth. Looking at the formal structure of this expansion, one can identify the physical regimes where it is possible to safely stop the iteration by considering only the solutions given in Eqs. (D1) and (D2): either when $\tilde{k}_{m \neq 0}(\omega) \ll \tilde{k}_{0}(\omega)$, namely a perturbation around the static term $\tilde{k}_{0}(\omega)$, or conversely, at high driving frequencies $\Omega$.

In the following we apply this scheme. We start by considering the perturbative expansions of $g_{v}(t)$ given in Eqs. (46) with Fourier transforms

$$
\begin{aligned}
& g_{n, 1}=\frac{1}{2}\left[\delta_{n, 1}+\delta_{n,-1}\right] \\
& g_{n, 2}=\sqrt{\frac{\gamma}{\gamma_{2}}}\left[\left(1-\frac{\kappa}{4}\right) \delta_{n, 0}-\frac{\kappa}{8}\left(\delta_{n, 2}+\delta_{n,-2}\right)\right]+\mathcal{O}\left(\kappa^{2}\right) .
\end{aligned}
$$

With these functions, the kernels $\tilde{k}_{n}(\omega)$ in Eq. (38) become

$$
\begin{aligned}
\tilde{k}_{n}(\omega) & =-i \sum_{\nu=1}^{2} \gamma_{\nu} \sum_{m=-\infty}^{+\infty} g_{m, \nu} g_{n-m, \nu}(\omega+m \Omega) \\
& =-i \gamma \omega \delta_{n, 0}+\mathcal{O}\left(\kappa^{2}\right) .
\end{aligned}
$$

We now apply the iterative solutions in Eqs. (D1)-(D2), obtaining the following $\kappa$ expansion of the Floquet coefficients:

$$
\tilde{G}_{m}(\omega)=\chi_{0}(\omega) \delta_{m, 0}+\mathcal{O}\left(\kappa^{2}\right) .
$$

Note that this expression fulfills the constraint of Eq. (42) up to linear order in $\kappa$. For this reason, we can directly use the general expressions (43) for the heat currents, valid in the constrained case, by inserting Eqs. (D3) and (D5).

We start with $J_{1}$. We observe the presence of the factor $\gamma_{1}=\kappa \gamma$ in front of the integral; this implies evaluating all other terms at zeroth order in $\kappa$, namely putting $v_{1}=2$ and $g_{n, 2}=\sqrt{\left(\gamma / \gamma_{2}\right)} \delta_{n, 0}$. The result is

$$
\begin{aligned}
J_{1}= & \kappa \gamma \int_{-\infty}^{+\infty} \frac{d \omega}{4 \pi}\left\{-\left(\omega^{2}+\Omega^{2}\right) \operatorname{Im} \chi_{0}(\omega) \operatorname{coth}\left(\frac{\omega}{2 T_{2}}\right)\right. \\
& \left.+\omega^{2} \operatorname{Im} \chi_{0}(\omega+\Omega) \operatorname{coth}\left(\frac{\omega}{2 T_{1}}\right)\right\}+\mathcal{O}\left(\kappa^{2}\right),
\end{aligned}
$$

as quoted in Eq. (47).

More cumbersome is the evaluation of $J_{2}$. Here, the first term in Eq. (43), called $J_{2}^{(a)}$, is

$$
\begin{aligned}
J_{2}^{(a)}= & \gamma\left(1-\frac{\kappa}{2}\right) \int_{-\infty}^{+\infty} \frac{d \omega}{2 \pi} \omega^{2} \operatorname{Im} \chi_{0}(\omega) \operatorname{coth}\left(\frac{\omega}{2 T_{2}}\right) \\
& +\mathcal{O}\left(\kappa^{2}\right),
\end{aligned}
$$

and it also contains a zeroth order term that, as we will see shortly, will be canceled out from the remaining part of $J_{2}$. This one, called $J_{2}^{(b)}$, receives contributions from both reservoirs $v_{1}=1,2$. For $v_{1}=1$, we again have to put $g_{n, 2}=\sqrt{\left(\gamma / \gamma_{2}\right)} \delta_{n, 0}$, obtaining

$$
\begin{aligned}
J_{2}^{(b)}\left(v_{1}=1\right)= & -\gamma \frac{\kappa}{2} \int_{-\infty}^{+\infty} \frac{d \omega}{2 \pi} \omega(\omega+\Omega) \operatorname{coth}\left(\frac{\omega}{2 T_{1}}\right) \\
& \times \operatorname{Im} \chi_{0}(\omega+\Omega)+\mathcal{O}\left(\kappa^{2}\right) .
\end{aligned}
$$

For $v_{1}=2$, the contribution $J_{2}^{(b)}\left(v_{1}=2\right)$ also has a zero term (opposite to that of $J_{2}^{(a)}$ ) in addition to the linear one:

$$
\begin{aligned}
J_{2}^{(b)}\left(v_{1}=2\right)= & -\gamma(1-\kappa) \int_{-\infty}^{+\infty} \frac{d \omega}{2 \pi} \omega^{2} \operatorname{coth}\left(\frac{\omega}{2 T_{2}}\right) \\
& \times \operatorname{Im} \chi_{0}(\omega)+\mathcal{O}\left(\kappa^{2}\right) .
\end{aligned}
$$


Summing up all these terms, we arrive at $J_{2}=J_{2}^{(a)}+$ $J_{2}^{(b)}\left(v_{1}=1\right)+J_{2}^{(b)}\left(v_{1}=2\right)$ with

$$
\begin{aligned}
J_{2}= & \kappa \gamma \int_{-\infty}^{+\infty} \frac{d \omega}{4 \pi}\left\{\omega^{2} \operatorname{Im} \chi_{0}(\omega) \operatorname{coth}\left(\frac{\omega}{2 T_{2}}\right)\right. \\
& \left.-\omega(\omega+\Omega) \operatorname{Im} \chi_{0}(\omega+\Omega) \operatorname{coth}\left(\frac{\omega}{2 T_{1}}\right)\right\}+\mathcal{O}\left(\kappa^{2}\right),
\end{aligned}
$$

which is the result quoted in Eq. (48).

We now present the explicit evaluation of expressions (D6)-(D10). First, we observe that $\chi_{0}(\omega)$ in Eq. (A18) can be decomposed as

$$
\chi_{0}(\omega)=-\frac{1}{2 \xi}\left[\frac{1}{\omega+i \lambda_{1}}-\frac{1}{\omega+i \lambda_{2}}\right]
$$

where

$$
\begin{aligned}
\lambda_{1} & =\frac{\gamma}{2}+i \xi, \quad \lambda_{2}=\frac{\gamma}{2}-i \xi \\
\xi & =\sqrt{\omega_{0}^{2}-\gamma^{2} / 4} .
\end{aligned}
$$

Note that, for $\gamma<2 \omega_{0}$, the roots are complex conjugate (underdamped oscillator); otherwise, for $\gamma>2 \omega_{0}$, they are real (overdamped oscillator). Hereafter, we consider the first case, which is the most interesting in the situation under investigation. In this regime, we have

$$
\begin{aligned}
& \operatorname{Im} \chi_{0}(\omega) \\
& \quad=\frac{\gamma}{4 \xi}\left[\frac{1}{\left(\omega+i \lambda_{1}\right)\left(\omega-i \lambda_{2}\right)}-\frac{1}{\left(\omega+i \lambda_{2}\right)\left(\omega-i \lambda_{1}\right)}\right] .
\end{aligned}
$$

Note that in the integrals for the average heat currents, Eqs. (47)-(48), the function $\operatorname{coth}\left(\omega / 2 T_{\nu}\right)$ is always present, which we now express as a series in the Matsubara frequencies $\omega_{n, v}=2 \pi n T_{\nu}$ :

$$
\operatorname{coth}\left(\frac{\omega}{2 T_{v}}\right)=2 T_{\nu}\left[\frac{1}{\omega}+2 \omega \sum_{n=1}^{+\infty} \frac{1}{\omega^{2}+\omega_{n, v}^{2}}\right] .
$$

The integration will be performed in the complex plane using the Cauchy method and considering a closed contour in the upper half plane. The poles are of two kinds: those given by the $\operatorname{Im} \chi_{0}(\omega)$ and $\operatorname{Im} \chi_{0}(\omega+\Omega)$, located at $\omega=$ $i \lambda_{2}, i \lambda_{1}$ and at $\omega=i \lambda_{2}-\Omega, i \lambda_{1}-\Omega$; and those given by the coth function, located at $\omega=i \omega_{n, v}$. Note that there is no pole in $\omega=0$. Moreover, since in general we are dealing with two different temperatures $T_{1}$ and $T_{2}$, the associated poles will be placed in different positions and one should properly take care of this fact while considering the limit procedure $\rho \rightarrow \infty$ of the radius of the closed path. We can then always split the result of integral (47) into a sum of the contribution due the poles of $\operatorname{Im} \chi_{0}$ and the contribution due to the poles of the coth function. Below, we explicitly evaluate $J_{1}$ and the average total power $P=-\left(J_{1}+J_{2}\right)$ from which one can also infer $J_{2}$. We can write

$$
\begin{aligned}
J_{1} & =J_{1, \chi}+J_{1, \mathrm{coth}}, \\
P & =P_{\chi}+P_{\text {coth }} .
\end{aligned}
$$

The contributions of the poles of $\operatorname{Im} \chi_{0}$ are

$$
\begin{aligned}
J_{1, \chi}= & \frac{\gamma \kappa}{8 \xi}\left\{\left(\Omega^{2}-\lambda_{1}^{2}\right) \operatorname{coth}\left(\frac{i \lambda_{1}}{2 T_{2}}\right)-\left(\Omega^{2}-\lambda_{2}^{2}\right)\right. \\
& \times \operatorname{coth}\left(\frac{i \lambda_{2}}{2 T_{2}}\right)+\left(i \lambda_{2}-\Omega\right)^{2} \operatorname{coth}\left(\frac{i \lambda_{2}-\Omega}{2 T_{1}}\right) \\
& \left.-\left(i \lambda_{1}-\Omega\right)^{2} \operatorname{coth}\left(\frac{i \lambda_{1}-\Omega}{2 T_{1}}\right)\right\}
\end{aligned}
$$

and

$$
\begin{aligned}
P_{\chi}= & -\frac{\gamma \kappa \Omega}{8 \xi}\left\{\Omega \operatorname{coth}\left(\frac{i \lambda_{1}}{2 T_{2}}\right)-\Omega \operatorname{coth}\left(\frac{i \lambda_{2}}{2 T_{2}}\right)\right. \\
& -\left(i \lambda_{2}-\Omega\right) \operatorname{coth}\left(\frac{i \lambda_{2}-\Omega}{2 T_{1}}\right) \\
& \left.+\left(i \lambda_{1}-\Omega\right) \operatorname{coth}\left(\frac{i \lambda_{1}-\Omega}{2 T_{1}}\right)\right\} .
\end{aligned}
$$

The poles of the coth function instead give the contributions

$$
\begin{aligned}
J_{1, \text { coth }}= & -i \gamma \kappa\left\{\sum_{n=1}^{+\infty} T_{2}\left(\Omega^{2}-\omega_{n, 2}^{2}\right) \operatorname{Im} \chi_{0}\left(i \omega_{n, 2}\right)\right. \\
& \left.+\sum_{n=1}^{+\infty} T_{1} \omega_{n, 1}^{2} \operatorname{Im} \chi_{0}\left(i \omega_{n, 1}+\Omega\right)\right\}
\end{aligned}
$$

and

$$
\begin{aligned}
P_{\text {coth }}= & i \gamma \kappa \Omega\left\{\sum_{n=1}^{+\infty} T_{2} \Omega \operatorname{Im} \chi_{0}\left(i \omega_{n, 2}\right)\right. \\
& \left.+\sum_{n=1}^{+\infty} i T_{1} \omega_{n, 1} \operatorname{Im} \chi_{0}\left(i \omega_{n, 1}+\Omega\right)\right\} .
\end{aligned}
$$

We are then left to resume the above series. This can be done thanks to the relatively simple form of $\operatorname{Im} \chi(\omega)$. The 
typical series we need is

$$
L_{a, b}=\sum_{n=1}^{+\infty} \frac{1}{(n+a)(n+b)}=\frac{1}{b-a}[\psi(b)-\psi(a)]-\frac{1}{a b}
$$

with $\psi(x)$ the digamma function. After a long but standard procedure we obtain

$$
\begin{aligned}
J_{1, \mathrm{coth}}= & i \frac{\gamma^{2} \kappa}{4 \xi}\left\{\frac{\Omega^{2}}{4 \pi^{2} T_{2}}\left[L_{a, b}-L_{c, d}\right]-T_{2} a^{2} L_{a, b}+T_{2} c^{2} L_{c, d}\right. \\
& +T_{1} \bar{a}^{2} L_{\bar{a}, \bar{b}}-T_{1} \bar{c}^{2} L_{\bar{c}, \bar{d}}+\frac{\Omega \xi}{\pi^{2} T_{1}} L_{\bar{b}, \bar{d}} \\
& \left.+\frac{i \xi}{\pi}\left[(\bar{b}-b) L_{b, \bar{b}}+(\bar{d}-d) L_{d, \bar{d}}+2 \log \left(T_{1} / T_{2}\right)\right]\right\}
\end{aligned}
$$

and

$$
\begin{aligned}
P_{\text {coth }}= & -\frac{\gamma^{2} \kappa}{4 \xi}\left\{\frac{i \Omega^{2}}{4 \pi^{2} T_{2}}\left[L_{a, b}-L_{c, d}\right]+\right. \\
& \left.-\frac{\Omega}{2 \pi}\left[(\bar{d}-\bar{b}) L_{\bar{b}, \bar{d}}-\bar{a} L_{\bar{a}, \bar{b}}+\bar{c} L_{\bar{c}, \bar{d}}\right]\right\},
\end{aligned}
$$

where, for notational convenience, we have defined the quantities

$$
\begin{array}{ll}
a=\frac{\lambda_{1}}{2 \pi T_{2}}, & \bar{a}=\frac{\lambda_{1}-i \Omega}{2 \pi T_{1}}, \\
b=\frac{-\lambda_{2}}{2 \pi T_{2}}, & \bar{b}=\frac{-\lambda_{2}-i \Omega}{2 \pi T_{1}}, \\
c=\frac{\lambda_{2}}{2 \pi T_{2}}, & \bar{c}=\frac{\lambda_{2}-i \Omega}{2 \pi T_{1}}, \\
d=\frac{-\lambda_{1}}{2 \pi T_{2}}, & \bar{d}=\frac{-\lambda_{1}-i \Omega}{2 \pi T_{1}} .
\end{array}
$$

\section{APPENDIX E: PERTURBATIVE REGIME FOR THE UNCONSTRAINED CASE}

In this appendix, we demonstrate that the unconstrained coupling fields considered in Eq. (51) belong to the same perturbative class as the constrained one.

Let us start by recalling the Fourier transforms of the coupling fields:

$$
\begin{aligned}
& g_{n, 1}=\frac{1}{2}\left[\delta_{n, 1}+\delta_{n,-1}\right], \\
& g_{n, 2}=\delta_{n, 0} .
\end{aligned}
$$

With these functions, the kernels $\tilde{k}_{n}(\omega)$ in Eq. (38) are exactly given by

$$
\begin{aligned}
\tilde{k}_{n}(\omega)= & -i \gamma_{2}\left[\omega\left(1+\frac{\kappa}{2}\right) \delta_{n, 0}+\frac{\kappa}{4}\left[(\omega+\Omega) \delta_{n, 2}\right.\right. \\
& \left.\left.+(\omega-\Omega) \delta_{n,-2}\right]\right],
\end{aligned}
$$

where we recall the definition of the effective asymmetry $\kappa=\gamma_{1} / \gamma_{2}$ and effective damping $\gamma=\operatorname{Max}\left[\gamma_{1}, \gamma_{2}\right]$. In the following we consider the perturbative regime $\kappa \rightarrow 0$. Inserting Eqs. (E2) and (E1) into the algebraic equations (31), the corresponding Floquet coefficients are

$$
\begin{aligned}
& \tilde{G}_{0}(\omega)=\chi_{0}(\omega)\left[1+i \frac{\omega \gamma \kappa}{2} \chi_{0}(\omega)\right]+\mathcal{O}\left(\kappa^{2}\right), \\
& \tilde{G}_{ \pm 2}(\omega)=i \frac{\gamma \kappa}{4}(\omega \pm \Omega) \chi_{0}(\omega \pm 2 \Omega) \chi_{0}(\omega)+\mathcal{O}\left(\kappa^{2}\right),
\end{aligned}
$$

$$
\tilde{G}_{|m|>2}(\omega)=\mathcal{O}\left(\kappa^{2}\right),
$$

with $\chi_{0}(\omega)=-1 /\left[\omega^{2}-\omega_{0}^{2}+i \gamma \omega\right]$ the bare susceptivity. Note that, for symmetry reasons, we always have $\tilde{G}_{2 m+1}(\omega)=0$. We now evaluate the general expressions (35) for the heat currents taking into account Eqs. (E1) and (E3). We start with $J_{1}$, which already contains the factor $\gamma_{1}=\kappa \gamma$ in front of the integral. This implies evaluating all other terms at zero order, namely, letting $\tilde{G}_{n_{1}+n_{2}}\left(\omega+n_{2} \Omega\right)=\chi_{0}\left(\omega+n_{2} \Omega\right) \delta_{n_{1},-n_{2}}$ in the first term on the right-hand side of Eq. (35) and letting $\tilde{G}_{m_{1}}\left(-\omega+n_{4} \Omega\right) \tilde{G}_{-\left(n_{\text {tot }}+m_{1}\right)}\left(\omega+n_{3} \Omega\right)=\chi_{0}(-\omega+$ $\left.n_{4} \Omega\right) \chi_{0}\left(\omega+n_{3} \Omega\right) \delta_{m_{1}, 0} \delta_{n_{\text {tot }}, 0}$ in the second term on the right-hand side of Eq. (35). With this procedure we obtain exactly the perturbative results of the constraint case given in Eq. (D6). We now consider $J_{2}$. The first term in Eq. (35), called $J_{2}^{(a)}$, has $n_{1}=n_{2}=0$ and can be written as

$$
J_{2}^{(a)}=-i \gamma \int_{-\infty}^{+\infty} \frac{d \omega}{2 \pi} \omega^{2} \operatorname{coth}\left(\frac{\omega}{2 T_{2}}\right) \tilde{G}_{0}(\omega) .
$$

Replacing $\tilde{G}_{0}(\omega)$ by the low- $\kappa$ expansion (E3a) we have

$$
\begin{aligned}
J_{2}^{(a)}= & \gamma \int_{-\infty}^{+\infty} \frac{d \omega}{2 \pi} \omega^{2} \operatorname{coth}\left(\frac{\omega}{2 T_{2}}\right) \\
& \times\left\{\operatorname{Im} \chi_{0}(\omega)+\frac{\omega \gamma \kappa}{2}\left[\Re^{2} \chi_{0}(\omega)-\operatorname{Im}^{2} \chi_{0}(\omega)\right]\right\} \\
& +\mathcal{O}\left(\kappa^{2}\right) .
\end{aligned}
$$

The second term, called $J_{2}^{(b)}$, has two contributions coming from both reservoirs $v_{1}=1,2$. For $v_{1}=1$, we again need to evaluate all terms at zero order in $\kappa$ because 
$\mathcal{J}_{\nu_{1}=1}(\omega)$ already contains $\gamma_{1}$. This implies that $m_{1}=0$, with $\tilde{G}_{0}(\omega)=\chi_{0}(\omega)$ and $n_{3}=-n_{4}$. The final expression for $J_{2}^{(b)}\left(v_{1}=1\right)$ is then equal to that quoted in Eq. (D8). We are left to consider the last term with $v_{1}=2$, called $J_{2}^{(b)}\left(v_{1}=2\right)$. Here, we have $n_{1}=n_{2}=n_{3}=n_{4}=0$ and up to linear order in $\kappa, m_{1}=0$ also. The result is

$$
\begin{aligned}
J_{2}^{(b)}\left(v_{1}=2\right)= & -\gamma^{2} \int_{-\infty}^{+\infty} \frac{d \omega}{2 \pi} \omega^{3} \operatorname{coth}\left(\frac{\omega}{2 T_{2}}\right)\left|\tilde{G}_{0}(\omega)\right|^{2} \\
& +\mathcal{O}\left(\kappa^{2}\right)
\end{aligned}
$$

By replacing

$$
\gamma \omega\left|\tilde{G}_{0}(\omega)\right|^{2}=\operatorname{Im} \chi_{0}(\omega)\left[1-\omega \gamma \kappa \operatorname{Im} \chi_{0}(\omega)\right]+\mathcal{O}\left(\kappa^{2}\right)
$$

we obtain

$$
\begin{aligned}
J_{2}^{(b)}\left(v_{1}=2\right)= & -\gamma \int_{-\infty}^{+\infty} \frac{d \omega}{2 \pi} \omega^{2} \operatorname{coth}\left(\frac{\omega}{2 T_{2}}\right) \operatorname{Im} \chi_{0}(\omega) \\
& \times\left\{1-\gamma \kappa \omega \operatorname{Im} \chi_{0}(\omega)\right\}+\mathcal{O}\left(\kappa^{2}\right) .
\end{aligned}
$$

Summing up all these terms we have $J_{2}=J_{2}^{(a)}+J_{2}^{(b)}\left(v_{1}=\right.$ 1) $+J_{2}^{(b)}\left(v_{1}=2\right)$, which is equal to the perturbative result (D10) obtained in the constrained case.

[1] G. Benenti, G. Casati, D. Rossini, and G. Strini, Principles of Quantum Computation and Information (A Comprehensive Textbook) (World Scientific, Singapore, 2019).

[2] M. Esposito, U. Harbola, and S. Mukamel, Nonequilibrium fluctuations, fluctuation theorems, and counting statistics in quantum systems, Rev. Mod. Phys. 81, 1665 (2009).

[3] M. Campisi, P. Hänggi, and P. Talkner, Colloquium: Quantum fluctuation relations: Foundations and applications, Rev. Mod. Phys. 83, 771 (2011).

[4] R. Kosloff, Quantum thermodynamics: A dynamical Viewpoint, Entropy 15, 2100 (2013).

[5] D. Gelbwaser-Klimovsky, W. Niedenzu, and G. Kurizki, Thermodynamics of quantum systems under dynamical control, Adv. At. Mol. Opt. Phys. 64, 329 (2015).

[6] S. Vinjanampathy and J. Anders, Quantum thermodynamics, Contemp. Phys. 57, 1 (2016).

[7] B. Sothmann, R. Sánchez, and A. N. Jordan, Thermoelectric energy harvesting with quantum dots, Nanotechnology 26, 032001 (2015).

[8] J. Goold, M. Huber, A. Riera, L. del Rio, and P. Skrzypczyk, The role of quantum information in thermodynamics-a topical review, J. Phys. A Math. Theor. 49, 1430001 (2016).

[9] G. Benenti, G. Casati, K. Saito, and R. S. Whitney, Fundamental aspects of steady-state conversion of heat to work at the nanoscale, Phys. Rep. 694, 1 (2017).
[10] P. Talkner and P. Hänggi, Colloquium: Statistical mechanics and thermodynamics at strong coupling: Quantum and classical, Rev. Mod. Phys. 92, 41002 (2020).

[11] G. T. Landi and M. Paternostro, arXiv:2009.07668 [quant$\mathrm{ph}]$.

[12] G. T. Landi, D. Poletti, and G. Schaller, arXiv:2104.14350 [quant-ph].

[13] F. Ciccarello, S. Lorenzo, V. Giovannetti, and G. M. Palma, arXiv:2106.11974 [quant-ph].

[14] N. Li, J. Ren, L. Wang, G. Zhang, P. Hänggi, and B. Li, Colloquium: Phononics: Manipulating heat flow with electronic analogs and beyond, Rev. Mod. Phys. 84, 1045 (2012).

[15] G. Benenti, G. Casati, C. Mejia-Monasterio, and M. Peyrard, in Thermal transport in low dimensions, S. Lepri (Ed.), Lecture Notes in Physics 921 (Springer, 2016).

[16] A. Fornieri and F. Giazotto, Towards phase-coherent caloritronics in superconducting circuits, Nat. Nanotech. 12, 944 (2017).

[17] J. P. Pekola and B. Karimi, arXiv:2107.12936 [condmat.mes-hall].

[18] G. Benenti, K. Saito, and G. Casati, Thermodynamic Bounds on Efficiency for Systems with Broken TimeReversal Symmetry, Phys. Rev. Lett. 106, 230602 (2011).

[19] A. E. Allahverdyan, K. V. Hovhannisyan, A. V. Melkikh, and S. G. Gevorkian, Carnot Cycle at Finite Power: Attainability of Maximal Efficiency, Phys. Rev. Lett. 111, 050601 (2013).

[20] R. S. Whitney, Most Efficient Quantum Thermoelectric at Finite Power Output, Phys. Rev. Lett. 112, 130601 (2014).

[21] M. F. Ludovico, J. S. Lim, M. Moskalets, L. Arrachea, and D. Sanchez, Dynamical energy transfer in ac-driven quantum systems, Phys. Rev. B 89, 161306 (2014).

[22] N. Shiraishi, K. Saito, and H. Tasaki, Universal Trade-Off Relation between Power and Efficiency for Heat Engines, Phys. Rev. Lett. 117, 190601 (2016).

[23] M. Campisi and R. Fazio, The power of a critical heat engine, Nat. Commun. 7, 11895 (2016).

[24] M. Polettini and M. Esposito, Carnot efficiency at divergent power output, Europhys. Lett. 118, 40003 (2017).

[25] P. Pietzonka and U. Seifert, Universal Trade-Off between Power, Efficiency, and Constancy in Steady-State Heat Engines, Phys. Rev. Lett. 120, 190602 (2018).

[26] R. Luo, G. Benenti, G. Casati, and J. Wang, Thermodynamic Bound on Heat-To-Power Conversion, Phys. Rev. Lett. 121, 080602 (2018).

[27] V. Holubec and A. Ryabov, Cycling Tames Power Fluctuations near Optimum Efficiency, Phys. Rev. Lett. 121, 120601 (2018).

[28] G. Benenti, G. Casati, and J. Wang, Power, efficiency, and fluctuations in steady-state heat engines, Phys. Rev. E 102, 040103(R) (2020).

[29] L. M. Cangemi, V. Cataudella, G. Benenti, M. Sassetti, and G. De Filippis, Violation of thermodynamics uncertainty relations in a periodically driven work-to-work converter from weak to strong dissipation, Phys. Rev. B 102, 165418 (2020).

[30] L. M. Cangemi, M. Carrega, A. De Candia, V. Cataudella, G. De Filippis, M. Sassetti, and G. Benenti, Optimal energy conversion through antiadiabatic driving 
breaking time-reversal symmetry, Phys. Rev. Res. 3, 013237 (2021).

[31] M. O. Scully, K. R. Chapin, K. E. Dorfman, M. B. Kim, and A. Svidzinsky, Quantum heat engine power can be increased by noise-induced coherence, Proc. Natl. Acad. Sci. U.S.A. 108, 15097 (2011).

[32] R. Uzdin, A. Levy, and R. Kosloff, Equivalence of Quantum Heat Machines, and Quantum-Thermodynamic Signatures, Phys. Rev. X 5, 031044 (2015).

[33] J. Klatzow, J. N. Becker, P. M. Ledingham, C. Weinzetl, K. T. Kaczmarek, D. J. Saunders, J. Nunn, I. A. Walmsley, R. Uzdin, and E. Poem, Experimental Demonstration of Quantum Effects in the Operation of Microscopic Heat Engines, Phys. Rev. Lett. 122, 110601 (2019).

[34] C. L. Latune, I. Sinayskiy, and F. Petruccione, ArXiv:1910. 14020 (2019).

[35] G. Watanabe, B. P. Venkatesh, P. Talkner, and A. del Campo, Quantum Performance of Thermal Machines Over Many Cycles, Phys. Rev. Lett. 118, 050601 (2017).

[36] K. Brandner, M. Bauer, and U. Seifert, Universal Coherence-Induced Power Losses of Quantum Heat Engines in Linear Response, Phys. Rev. Lett. 119, 170602 (2017).

[37] M. Carrega, M. Sassetti, and U. Weiss, Optimal work-towork conversion of a nonlinear quantum Brownian duet, Phys. Rev. A 99, 062111 (2019).

[38] K. Brandner and K. Saito, Thermodynamic Geometry of Microscopic Heat Engines, Phys. Rev. Lett. 124, 040602 (2020).

[39] G. Francica, F. C. Binder, G. Guarnieri, M. T. Mitchison, J. Goold, and F. Plastina, Quantum Coherence and Ergotropy, Phys. Rev. Lett. 125, 180603 (2020).

[40] A. Levy, R. Alicki, and R. Kosloff, Quantum refrigerators and the third law of thermodynamics, Phys. Rev. E 85, 061126 (2012).

[41] G. Benenti and G. Strini, Dynamical Casimir effect and minimal temperature in quantum thermodynamics, Phys. Rev. A 91, 020502(R) (2015).

[42] N. Freitas and J. P. Paz, Fundamental limits for cooling of linear quantum refrigerators, Phys. Rev. E 95, 012146 (2017).

[43] N. Freitas and J. P. Paz, Cooling a quantum oscillator: A useful analogy to understand laser cooling as a thermodynamical process, Phys. Rev. A 97, 032104 (2018).

[44] F. Clivaz, R. Silva, G. Haack, J. B. Brask, N. Brunner, and M. Huber, Unifying Paradigms of Quantum Refrigeration: A Universal and Attainable Bound on Cooling, Phys. Rev. Lett. 123, 170605 (2019).

[45] F. Giazotto, T. T. Heikkilä, A. Luukanen, A. M. Savin, and J. P. Pekola, Opportunities for mesoscopics in thermometry and refrigeration: Physics and applications, Rev. Mod. Phys. 78, 217 (2006).

[46] M. J. Martínez-Pérez and F. Giazotto, A quantum diffractor for thermal flux, Nat. Commun. 5, 3579 (2014).

[47] H. J. D. Miller, M. Scandi, J. Anders, and M. PerarnauLLobet, Work Fluctuations in Slow Processes: Quantum Signatures and Optimal Control, Phys. Rev. Lett. 123, 230603 (2019).

[48] P. Abiuso and M. Perarnau-LLobet, Optimal Cycles for Low-Dissipation Heat Engines, Phys. Rev. Lett. 124, 110606 (2020).
[49] N. Pancotti, M. Scandi, M. T. Mitchison, and M. PerarnauLLobet, Speed-Ups to Isothermality: Enhanced Quantum Thermal Machines through Control of the System-Bath Coupling, Phys. Rev. X 10, 031015 (2020).

[50] H.-P. Breuer and F. Petruccione, The Theory of Open Quantum Systems (Oxford University Press, New York, 2002).

[51] U. Weiss, Quantum Dissipative Systems (World Scientific, Singapore, 2012).

[52] J. Iles-Smith, N. Lambert, and A. Nazir, Environmental dynamics, correlations, and the emergence of noncanonical equilibrium states in open quantum systems, Phys. Rev. A 90, 032114 (2014).

[53] H.-P. Breuer, E.-M. Laine, J. Piilo, and B. Vacchini, Colloquium: Non-Markovian dynamics in open quantum systems, Rev. Mod. Phys. 88, 021002 (2016).

[54] I. de Vega and D. Alonso, Dynamics of non-markovian open quantum systems, Rev. Mod. Phys. 89, 015001 (2017).

[55] M. Carrega, P. Solinas, A. Braggio, M. Sassetti, and U. Weiss, Functional integral approach to time-dependent heat exchange in open quantum systems: General method and applications, New J. Phys. 17, 045030 (2015).

[56] L. Henriet, Z. Ristivojevic, P. P. Orth, and K. Le Hur, Quantum dynamics of the driven and dissipative rabi model, Phys. Rev. A 90, 023820 (2014).

[57] E. Aurell, Characteristic functions of quantum heat with baths at different temperatures, Phys. Rev. E 97, 062117 (2018).

[58] P. Menczel, E. Loisa, K. Brandner, and C. Flindt, Thermodynamic uncertainty relations for coherently driven open quantum systems, J. Phys. A: Math. Theor. 54, 314002 (2021).

[59] I. Khait, J. Carrasquilla, and D. Segal, ArXiv:2108.12441 (2021).

[60] A. Riera-Campeny, A. Sanpera, and P. Strasberg, Quantum systems correlated with a finite bath: Nonequilibrium dynamics and Thermodynamics, PRX Quantum 2, 010340 (2021).

[61] M. Wiedmann, J. T. Stockburger, and J. Ankerhold, NonMarkovian dynamics of a quantum heat engine: out-ofequilibrium operation and thermal coupling control, New J. Phys. 22, 033007 (2020).

[62] M. M. Müller, R. S. Said, F. Jelezko, T. Calarco, and S. Montangero, ArXiv:2104.07687 (2021).

[63] F. Zhan, N. Li, S. Kohler, and P. Hänggi, Molecular wires acting as quantum heat ratchets, Phys. Rev. E 80, 061115 (2009).

[64] N. Li, P. Hänggi, and B. Li, Ratcheting heat flux against a thermal bias, Eur. Phys. Lett. 84, 40009 (2008).

[65] A. O. Caldeira and A. J. Leggett, Quantum tunnelling in a dissipative system, Ann. Phys. 149, 374 (1983).

[66] C. Zherbe and P. Hanggi, Brownian parametric quantum oscillator with dissipation, Phys. Rev. E 52, 1533 (1995), Phys. Rev. B.

[67] A. Schnirman, Y. Makhlin, and G. Schoen, Noise and decoherence in quantum two-level Systems, Physica Scripta T102, 147 (2002).

[68] M. H. Devoret and R. J. Schoelkopf, Superconducting circuits for quantum information: An Outlook, Science 339, 1169 (2013). 
[69] We denote instead as $O(t)$ an observable $O$ at time $t$ in the Heisenberg picture.

[70] A. Blais, R. Huang, A. Wallraff, S. M. Girvin, and R. J. Schoelkopf, Cavity quantum electrodynamics for superconducting electrical circuits: An architecture for quantum computation, Phys. Rev. A 69, 062320 (2004).

[71] P. Strasberg, G. Schaller, T. Brandes, and M. Esposito, Quantum and Information Thermodynamics: A Unifying Framework Based on Repeated Interactions, Phys. Rev. X 7, 021003 (2017).

[72] We note that the definition of time-dependent heat current in general should be considered with care $[42,61$, 71,115-117]. Indeed, there are nontrivial contributions that may arise from the Hamiltonian that describe the interaction between the system and bath, especially in the case of strong coupling [71]. However, these additional, time-dependent contributions vanish once averaged over a cycle and thus we can safely use the definition in Eq. (19).

[73] H. B. Callen, Thermodynamics and an Introduction to Thermostatistics (John Wiley \& Sons, New York, 1985).

[74] D. Kondepudi and I. Prigogine, Modern Thermodynamics: from Heat Engines to Dissipative Structures (John Wiley \& Sons, Singapore, 2014).

[75] L. Arrachea, E. Mucciolo, C. Chamon, and R. Capaz, Microscopic model of a phononic refrigerator, Phys. Rev. B 86, 125424 (2012).

[76] M. Grifoni, M. Sassetti, P. Hanggi, and U. Weiss, Cooperative effects in the nonlinearly driven spin-boson system, Phys. Rev. E 52, 3596 (1995).

[77] M. Grifoni, M. Sassetti, and U. Weiss, Exact master equations for driven dissipative tight-binding models, Phys. Rev. E 53, R2033 (1996).

[78] A. Riera-Campeny, M. Mehboudi, M. Pons, and A. Sanpera, Dynamically induced heat rectification in quantum systems, Phys. Rev. E 99, 032126 (2019).

[79] N. Li, F. Zhan, P. Hänggi, and B. Li, Shuttling heat across one-dimensional homogenous nonlinear lattices with a Brownian heat motor, Phys. Rev. E 80, 011125 (2009).

[80] C. de Tomas, A. C. Hernandez, and J. M. M. Roco, Optimal low symmetric dissipation carnot engines and refrigerators, Phys. Rev. E 85, 010104(R) (2012).

[81] A. E. Allahverdyan, K. Hovhannisyan, and G. Mahler, Optimal refrigerator, Phys. Rev. E 81, 051129 (2010).

[82] F. Vischi, M. Carrega, E. Strambini, P. Virtanen, A. Braggio, and F. Giazotto, Thermodynamic cycles in josephson junctions, Sci. Rep. 9, 3238 (2019).

[83] V. Sing, T. Pandit, and R. S. Johal, Optimal performance of a three-level quantum refrigerator, Phys. Rev. E 101, 062121 (2020).

[84] B. Bhandari, R. Fazio, F. Taddei, and L. Arrachea, From nonequilibrium green's functions to quantum master equations for the density matrix and out-of-time-order correlators: Steady-state and adiabatic dynamics, Phys. Rev. B 104, 035425 (2021).

[85] L. Vannucci, F. Ronetti, G. Dolcetto, M. Carrega, and M. Sassetti, Interference-induced thermoelectric switching and heat rectification in quantum Hall junctions, Phys. Rev. B 92, 075446 (2015).
[86] D. Segal and A. Nitzan, Spin-Boson Thermal Rectifier, Phys. Rev. Lett. 94, 034301 (2005).

[87] R. Sanchez, H. Thierschmann, and L. W. Molenkamp, Single-electron thermal devices coupled to a mesoscopic gate, New J. Phys. 19, 113040 (2017).

[88] A. Marcos-Vicioso, C. Lopez-Jurado, M. Ruiz-Garcia, and R. Sanchez, Thermal rectification with interacting electronic channels: Exploiting degeneracy, quantum superpositions, and interference, Phys. Rev. B 98, 035414 (2018).

[89] R. Sanchez, C. Gorini, and G. Fleury, ArXiv:2107.06723 (2021).

[90] L. Bours, B. Sothmann, M. Carrega, E. Strambini, A. Braggio, E. M. Hankiewicz, L. W. Molenkamp, and F. Giazotto, Phase-Tunable Thermal Rectification in the Topological SQUIPT, Phys. Rev. Appl. 11, 044073 (2019).

[91] T. Motz, M. Wiedmann, J. T. Stockburger, and J. Ankerhold, Rectification of heat currents across nonlinear quantum chains: a versatile approach beyond weak thermal contact, J. Phys. 20, 113020 (2018).

[92] B. Bhandari, P. A. Erdman, R. Fazio, E. Paladino, and F. Taddei, Thermal rectification through a nonlinear quantum resonator, Phys. Rev. B 103, 155434 (2021).

[93] P. Portugal, C. Flindt, and N. Lo Gullo, ArXiv:2103.07114 (2021).

[94] M. Acciai, F. Hajiloo, F. Hassler, and J. Splettstoesser, Phase-coherent heat circulators with normal or superconducting contacts, Phys. Rev. B 103, 085409 (2021).

[95] M. Peyrard, Comment on "dynamically induced heat rectification in quantum systems", Phys. Rev. E 101, 016101 (2020).

[96] R. Bosisio, S. Valentini, F. Mazza, G. Benenti, R. Fazio, V. Giovannetti, and F. Taddei, Magnetic thermal switch for heat management at the nanoscale, Phys. Rev. B 91, 205420 (2015).

[97] R. Sanchez, H. Thierschmann, and L. W. Molenkamp, All-thermal transistor based on stochastic switching, Phys. Rev. B 95, 241401 (2017).

[98] J. Yang, C. Elouard, J. Splettstoesser, B. Sothmann, R. Sanchez, and A. N. Jordan, Thermal transistor and thermometer based on coulomb-coupled conductors, Phys. Rev. B 100, 045418 (2019).

[99] G. Timossi, A. Fornieri, F. Paolucci, C. Puglia, and F. Giazotto, Phase-tunable josephson thermal Router, Nanolett. 18, 1764 (2018).

[100] F. Vischi, M. Carrega, E. Strambini, S. D’Ambrosio, F. S. Bergeret, Y. V. Nazarov, and F. Giazotto, Coherent transport properties of a three-terminal hybrid superconducting interferometer, Phys. Rev. B 95, 054504 (2017).

[101] J. P. Pekola and F. W. J. Hekking, Normal-MetalSuperconductor Tunnel Junction as a Brownian Refrigerator, Phys. Rev. Lett. 98, 210604 (2007).

[102] A. Mari and J. Eisert, Cooling by Heating: Very Hot Thermal Light Can Significantly Cool Quantum Systems, Phys. Rev. Lett. 108, 120602 (2012).

[103] B. Cleuren, B. Rutten, and C. Van den Broeck, Cooling by Heating: Refrigeration Powered by Photons, Phys. Rev. Lett. 108, 120603 (2012).

[104] J.-H. Jiang, O. Entin-Wohlman, and Y. Imry, Thermoelectric three-terminal hopping transport through 
one-dimensional nanosystems, Phys. Rev. B 85, 075412 (2012).

[105] R. Sánchez, B. Sothmann, and A. N. Jordan, Chiral Thermoelectrics with Quantum Hall Edge States, Phys. Rev. Lett. 114, 146801 (2015).

[106] F. Mazza, S. Valentini, R. Bosisio, G. Benenti, V. Giovannetti, R. Fazio, and F. Taddei, Separation of heat and charge currents for boosted thermoelectric conversion, Phys. Rev. B 91, 245435 (2015).

[107] V. Cavina, A. Mari, A. Carlini, and V. Giovannetti, Variational approach to the optimal control of coherently driven, open quantum system dynamics, Phys. Rev. A 98, 052125 (2018).

[108] G. Manzano, F. Plastina, and R. Zambrini, Optimal Work Extraction and Thermodynamics of Quantum Measurements and Correlations, Phys. Rev. Lett. 121, 120602 (2018).

[109] P. A. Erdman and F. Noe, ArXiv:2108.13525 (2021).

[110] J. Tuorila, J. T. Stockburger, T. Ala-Nissila, J. Ankerhold, and M. Mottonen, System-environment correlations in qubit initialization and control, Phys. Rev. Res. 1, 013004 (2019).
[111] M. Xu, J. T. Stockburger, and J. Ankerhold, Heat transport through a superconducting artificial atom, Phys. Rev. B 103, 104304 (2021).

[112] Y. Lu, N. Lambert, A. F. Kockum, K. Funo, A. Bengtsson, S. Gasparinetti, F. Nori, and P. Delsing, ArXiv:2107.12700 (2021).

[113] H. Leitch, N. Piccione, B. Bellomo, and G. De Chiara, ArXiv:2108.11341 (2021).

[114] A. R. Milne, C. Hempel, L. Li, C. L. Edmunds, H. J. Slatyer, H. Ball, M. R. Hush, and M. J. Biercuk, Quantum Oscillator Noise Spectroscopy via Displaced Cat States, Phys. Rev. Lett. 126, 250506 (2021).

[115] M. Esposito, M. Ochoa, and M. Galperin, Quantum Thermodynamics: A Nonequilibrium Green's Function Approach, Phys. Rev. Lett. 114, 080602 (2015).

[116] M. F. Ludovico, M. Moskalets, D. Sanchez, and L. Arrachea, Dynamics of energy transport and entropy production in ac-driven quantum electron systems, Phys. Rev. B 94, 035436 (2016).

[117] A. K. Slimane, P. Reck, and G. Fleury, Simulating timedependent thermoelectric transport in quantum systems, Phys. Rev. B 101, 235413 (2020). 